\title{
Evaluating Ambiguous Offerings
}

\author{
ROMAIN \\ BOULONGNE \\ IESE Business School \\ rboulongne@iese.edu
}

\author{
RODOLPHE \\ DURAND \\ HEC Paris \\ durand@hec.fr
}

\begin{abstract}
:
This paper studies how audience members categorize and evaluate ambiguous offerings. Depending on whether audience members categorize ambiguous offerings based on prototypes or goals, they activate two distinct cognitive mechanisms and evaluate differently ambiguous offerings. We expect that when audiences engage in goal-based vs. prototype-based categorization, their evaluation of ambiguous products increases. We theorize that under goal-based categorization, the perceived utility of unclear attributes increases for audiences, which leads them to evaluate more positively ambiguous product offerings. We test and find support for these direct and mediated relationships through a series of lab, on-line and field experiments. Overall, this study offers important implications for research on product and market categories, optimal distinctiveness, and market agents' cognitive ascription of value.
\end{abstract}

Forthcoming, Organization Science 
Market audiences tend to discount ambiguous products, i.e. products that disrespect established categories or present ill-defined attributes. Ambiguity means for audiences that they cannot associate an attribute, because it is unclear or because it is borrowed from a distinct category, with a corresponding product category (Fleischer, 2009). Audiences thereby call into question ambiguous products' identities and the competence of their producers (Hsu 2006, Hsu et al. 2009). However, despite the potential discount, producers routinely introduce ambiguous products that violate established market categories (Durand and Paolella 2013, Suarez et al. 2015, Zhao et al. 2018). Business press and academic research tout the recipe for creating new products: combine two completely separate categories, which leads to innovations such as a mobile phone that monitors the user's glucose level or a toaster that diffuses radio stations (Wall Street Journal, 2007; Gibbert et al, 2012). We need therefore to understand the chiasm between practice and theory, and more precisely the conditions that would make ambiguous products valuable for audiences.

Directly addressing this question and drawing on past research on product categories in markets (for reviews, see Vergne and Wry 2014, and Durand, Granqvist and Tyllstrom, 2017), our baseline hypothesis states that the default categorization is prototype-based and results in lower evaluations of an ambiguous product (Rosch and Mervis 1975, Hannan et al. 2007, Leung and Sharkey 2014). Indeed, audiences prefer well-identified products that possess the expected characteristics of their category. For instance, films and wines receive better evaluations when they match crisp categories, such as comedy or barolo (Hsu 2006, Negro et al. 2010). This perspective on categories assumes that audiences mainly categorize entities using stored product categories and search for instances that possess the attributes that best represent these categories (Hsu et al. 2009). We contend that this assumption does not characterize many market situations in which audiences have a particular need and categorize product offerings based on the goal they pursue-a categorization process called goal-based in the literature (Barsalou, 1985; Paolella and Durand, 
2016). Hence, we relax this assumption and theorize that when goal-based categorization is primed, audience members shift their attention away from a similarity check with a stored prototype to assess whether offered attributes can serve to achieve the goal at hand (Barsalou, 1983, 1987 \& 1991). If evaluators' primary focus is to achieve an ad hoc goal, i.e. a goal not usually associated with the typical functions of a prototype, they no longer exclusively assess the entity in relation to a stored prototype, but instead consider whether the entity is a good candidate to meet their goals (Durand and Boulongne, 2017). Under the minimal condition that there exists some association between the primed goal and the attributes of the product offering, we propose further that when goal-based categorization is primed (vs. prototype-based categorization), the evaluator activates a different cognitive mechanism (conceptual combination vs. central tendency) that establishes linkages between attributes and the pursued goal. Whereas central tendency results in a systematic discount of ambiguity, this is not the case when conceptual combination is active, and thus the evaluator is more likely to interpret favorably ambiguous products when goal-based categorization is primed (Pontikes 2012, Paolella and Durand 2016). More precisely, conceptual combination is more likely to ascribe a positive value to unclear attributes with respect to the pursued goal, which in turn explains why an evaluator values more favorably ambiguous products as she otherwise might under prototype-based categorization -reciprocally, priming goal-based categorization when evaluating non-ambiguous products may reduce their evaluation as central tendency is more efficient at identifying and valuing non-ambiguous products.

Therefore, we developed a series of experiments to test two main hypotheses: that goalbased activation is associated with a higher evaluation of ambiguous products $(\mathrm{H} 1)$ and that the perceived utility of unclear attributes is the mediator that drives this positive association (H2). Study 1 shows that whereas participants (320 M-Turkers) discount an ambiguous financial product when prototype-based categorization is primed, the priming of goal-based categorization increases their 
evaluation. Study 2 (with 317 M-Turkers) replicates this finding using a consumer good instead of a financial product. Study 2 provides evidence of the underlying cognitive mechanism at play: goalbased categorization increases the perceived utility of an unclear attribute, which in turn impacts positively the evaluation of the ambiguous product. In addition, we supplement both studies with four lab, field and on-line experiments (see on-line appendix).

Beyond filling a gap in category research by concurrently testing prototype-based and goalbased categorizations on the evaluation of an ambiguous product, these results have meaningful implications for research on organizations and practice. First, we redirect attention from the outcome of the evaluation to the process of evaluation (Pavelchak 1989, Bowers 2015, Smith and Chae, 2017), i.e. toward the cognitive mechanisms that audience members use to make sense of a producer's market offering (Grégoire et al. 2010, Kahl 2015). As such, by analyzing the connection between product attributes and evaluators' cognitive mechanisms, we complement existing research that remains disconnected from the evaluator and makes blanket assumptions about how audiences as a group use prototype- and goal-based categorization (e.g. Pontikes, 2012; Paolella and Durand, 2016). Second, this study contributes to explaining the conditions under which an organization and its products can differ but still be acceptable in the organization's industry (e.g., Jensen and Kim, 2014, Pontikes and Barnett, 2015, Zhao et al. 2017). Finally, this study provides the theoretical ground to better understand how audiences' cognitive interpretations of a producer's offerings affect market perception and competition (Anthony et al. 2016, Cattani et al. 2017).

\section{THEORY BACKGROUND AND HYPOTHESES DEVELOPMENT}

\section{Ambiguity and Evaluation}

Categories, by serving as the cognitive interface between producers and audiences (Rosa et al. 1999, Cattani et al. 2017), “represent a meaningful consensus about some entities' features as shared by actors grouped together as an audience" (Durand and Paolella 2013, p. 1100). Categories possess a 
strategic importance for firms to attract funding (Wry et al. 2014), impose their innovations

(Granqvist et al. 2013, Suarez et al. 2015), build their reputation (Benner and Tripsas 2012, Durand, Rao, Monin, 2007), and shape competition in markets (Anthony et al. 2016, Cattani et al. 2017).

One approach of market categories considers how category members relate to a prototype, the entity that best represents the category (Hannan et al. 2007, Zhao et al. 2018). Prototypes are specimens that possess all the coding clues of a specific category, such as a tactile screen, a digital camera, and an Internet connection for a mobile phone (Suarez et al. 2015). For this prototypebased approach, evaluators assess offerings based on a prototype stored in their memory; people use these crisp sets of attributes in their attempts to simplify a complex reality and ease their cognitive processing (Hannan et al. 2007). In the prototype-based approach, evaluators' central tendency is the default cognitive mechanism that identifies and evaluates entities by associating the entity's characteristics with the expected central features of the prototype (Barsalou 1985, Hannan et al. 2007). Judgments that mobilize central tendency are holistic, efficient, rapid, and effortless (Fiske and Pavelchak 1986; Pavelchak 1989). However, offerings that do not fit into an audience's prestored schemata lack appeal and pose cognitive difficulties. Hence, when features belong to other categories, contradict, or do not coincide with expected attributes, the offering appears ambiguous and is sanctioned (Fiske and Pavelchak 1986, Hannan et al 2007, Hsu et al. 2009).

In this context, the presence of one or several unclear attributes that are subject to multiple interpretations with respect to an existing product category generates ambiguity for the evaluator (Gregan-Paxton et al. 2005, Pontikes 2012). Audiences cannot easily process the information needed, both to understand where an offering stands in a category system and to retrieve the corresponding prototype (Rosch and Mervis 1975, Barsalou 1985). Hence, when a product possesses one or several imprecise attributes and audiences seek for prototypes, "ambiguity causes confusion and results in devaluation" (Pontikes, 2012, p. 84), as audiences cannot associate the unclear product 
attribute(s) with a single product category (Hannan et al. 2007), and question the identity and competence of producers (Hsu et al. 2009, Negro and Leung 2013).

\section{Ambiguity and Goal-Based Categorization}

A second perspective suggests that prototype-based categorization is not the only categorization process that audiences use in markets. The goal-based view of categories explains that audiences evaluate entities in terms of how they can meet one’s needs (Barsalou 1991, Durand and Boulongne, 2017, Durand and Paolella, 2013). For instance, Paolella and Durand (2016) found that when a corporate law firm offered multiple legal services, and hence exhibited potential ambiguity, the firm received more positive evaluations and performed better. That is, clients who pursued complex goals (e.g., making an acquisition) did not consider whether a corporate law firm was prototypical but rather whether it could manage the multiple demands implied by the acquisition. Furthermore, firms recursively use goal-based categorization in markets to ease an audience's understanding of new products, that is for offerings that are neither well established, nor stored in our memories, and for which there is no prototype (Tripsas 2009, Navis and Glynn 2010, Zhao et al. 2017). Thus, when industries are nascent, to make sense of ambiguity introduced by innovative product attributes, producers emphasize the goal that their new product can serve, rather than attempting to have the product fit with a prototype (Cusumano et al. 2015). In the mobile handset industry, Klingebiel and Joseph (2016) found that firms that moved early were more likely to offer a broad array of new features, which increased ambiguity. As an example, although touch screens gradually became a typical attribute of the smartphone category, they were unclear and not ubiquitous when the category emerged but ended to be preferred by customers (Suarez et al. 2015).

The favorable evaluation of ambiguity results from evaluators' primary focus to achieve an ad hoc goal, i.e. a goal not usually associated with the typical functions of a prototype. When evaluators consider whether an entity is a good candidate to meet their goal (under the obvious and 
necessary condition that there exists some minimal correspondence between the goal and some of the product attributes), audiences no longer exclusively assess the entity in relation to a stored prototype. They switch from activating central tendency, the default cognitive mechanism associated with prototype-based categorization, to activating conceptual combination, the cognitive mechanism associated with goal-based categorization (Barsalou 1991, Chrysikou 2006). As an illustration, Barsalou (1991; 47) explains that "someone might first categorize an entity as a chair but then categorize it subsequently into things that can be stood on to change a light bulb."

Authors have suggested—but have not tested—-that when evaluators' goals come first, their cognitive process passes from type- to goal-based, leading evaluators to positively ascribe value to ambiguous offerings (Durand and Paolella 2013, Paolella and Durand 2016, Zuckerman 2017). Since the product attributes are evaluated in correspondence with a goal—and not in relation to a prototype- the possibility to perceive a benefit in ambiguity with respect to the pursued goal is necessarily greater than when central tendency operates (in this latter case, ambiguity is systematically discounted.) Hence, because conceptual combination is a cognitive mechanism that establishes connections between attributes and a goal, the likelihood that an ambiguous product has the potential to serve a goal and is valued positively is higher than in the prototype-based categorization where it is systematically dismissed. When evaluating a non-ambiguous offering, however, the benefits of activating goal-based categorization on evaluation may not materialize. Indeed, goal-based categorization directs the evaluator's attention away from the standard and expected functions of the product, probing the different attributes with respect to the goal under consideration. This cognitive effort may prove more costly and less satisfactory than when evaluators rely on more efficient assessment provided by central tendency (Barsalou 1985, 1991, Fiske and Pavelchak 1986) and hence result in lower evaluations of non-ambiguous products. Overall, the baseline expectation is a negative evaluation of ambiguous products. However, 
when audiences activate conceptual combination and assess the offering in terms of meeting an ad hoc goal and not as fitting into an established prototype, their evaluation of an ambiguous product offering is more positive relatively to the baseline expectation, provided that the unclear attributes are at least minimally relevant to the goal at hand. Thus:

Hypothesis 1: Goal-based categorization leads to a positive evaluation of ambiguous products (relative to the baseline condition)

We reasoned that the likelihood to find an interest in product ambiguity is statistically greater when evaluators are primed with a goal than when they refer to existing prototypes. In the latter case, they simply tend to reject ambiguity. We go deeper and hypothesize that this average effect is due to the higher valuation of unclear attributes. When audiences rely on prototype-based categorization, evaluators seek attributes that are already established in the audience's mind, which limits the audience's capacity to see any utility in unclear product attributes (Barsalou, 1991, 1995, Ratneshwar et al. 2001). As such, when evaluating a chair, if not primed on an ad hoc goal (e.g. "change a light bulb”), one might discount an unclear attribute (say, disproportionally high legs that make it resemble a bar stool) — as it doesn't fit with standard expectations. In the context of goal pursuit however, having an unclear attribute such as disproportionally high legs might potentially be useful to the goal at hand (e.g., changing a light bulb), which leads evaluators to not instantly discount the entity and even assess it favorably because the presence of this unclear attribute becomes valuable. Likewise, whereas the utility of voice control to access internet is atypical and may not be perceived favorably (e.g. compared to typing questions and reading answers on a screen), home appliances like Google Home or Alexa become valued when evaluators are primed with a goal (e.g. simultaneously cooking and browsing the net).

Under goal-based categorization, conceptual combination puts to a test each offerings' attributes and value them in relation to the goal (Ratneshwar et al. 2001). Unclear attributes are no 
longer evaluated through the lens of a prototype but along multiple dimensions that are instrumental to the goal at hand (Gregan-Paxton et al. 2005, Pontikes, 2012). In general, when goal-based categorization is activated, evaluators focus on an offering's separate attributes, generate more attribute-related thoughts, and pay more attention to unclear attributes in order to achieve a specific goal (Gibbert and Mazursky 2009, Roy and Cornwell 2004, Paolella and Durand 2016). As a result of this methodical examination, Gibbert and Mazursky (2009) explain that as audiences engage in conceptual combination, they bear higher cognitive costs (relatively to central tendency which is quick, effortless, and similarity-based). Furthermore, Labroo and Kim (2009) show that there exists an association between the effort put in an evaluative task and the value attributed to it. Therefore, unclear attributes offer multiple potential interpretations in liaison with the pursued goal, are more cognitively demanding to process, and more likely to be rewarded. If this mechanism is at play, it accounts for the positive evaluation of unclear attributes but should not be manifest in the evaluation of clear attributes. ${ }^{1}$ Overall, if the activation of goal-based categorization leads to higher evaluation of ambiguous products, it is through the increase in the perceived utility of unclear attributes. Evaluators may perceive these attributes as being useful to achieve a certain goal and reward them more as they examine their utility in the first place (Labroo and Kim, 2009). Thus, goalbased categorization triggers an effortful cognitive process, and it is likely that this process positively impacts the perceived utility of unclear attributes, which leads to higher evaluation of ambiguous products (Barsalou 1991, Chrysikou 2006, Gibbert and Mazursky 2009). Hence, we hypothesize that: Hypothesis 2: The perceived utility of unclear product attributes mediates the positive effect of goal-based categorization on the evaluation of ambiguous products

\footnotetext{
${ }^{1}$ Labroo and Kim (2009) called this mechanism an "instrumentality heuristic". They tested it by manipulating the level of visual clarity of an advertisement on its evaluation: participants read either a blurry (difficult-to-process) or a clear (easy-to-process) advertisement and tended to value the former more.
} 


\section{OVERVIEW OF THE STUDIES}

Our research project uses a combination of online, lab and field experiments to investigate our hypotheses. Table 1 presents more details about the six studies that we run to test $\mathrm{H} 1$ and $\mathrm{H} 2$. We present two studies in the paper that we complement with four additional studies available in the online appendix. This research project was initiated because a large pubic bank was facing difficulties in promoting its loans and financial products to underserved entrepreneurs. We signed a research agreement with this bank ${ }^{2}$ and use a loan as a product in Study 1 (and studies A and B).

Study 1 uses a 2 (low versus high ambiguity) x 3 (control versus prototype-based versus goal-based) design to investigate whether the priming of goal-based categorization positively impacts the evaluation of an ambiguous offering (H1), hence here a loan. Study 2 employs the same design than Study 1 to replicate and complement the findings of our first study, this time using a consumer good instead of a financial product. Importantly, Study 2 introduces a novel measure that captures the perceived utility of each product attribute, and tests whether goal-based categorization impacts positively the evaluation of an unclear attribute (versus clear attributes) and whether the perceived utility of the unclear attribute accounts for the positive effect of goal-based categorization on the evaluation of the ambiguous offering (H2).

\section{Insert Table 1 about here}

As per Table 1, the online appendix contains four studies. Study A tests, in the lab, that when participants are placed in a control condition or primed with prototype-based categorization, they tend to discount an ambiguous offering (the loan as in Study 1) making central tendency the baseline categorization mechanism. Study B replicates the findings of Study 1 using a population of real-world entrepreneurs - the initial target of the loan.

\footnotetext{
2 "Public Bank" is a major public investment bank and has total assets of \$42 billion, including $\$ 20$ billion in loans, $\$ 12$ billion in guarantees, and $\$ 10$ billion in equity financing. At the time of our partnership, "Public Bank" was redesigning its online strategy to attract more underserved entrepreneurs — partly due to its ongoing struggle to identify which product attributes to present to its target beneficiaries on its website.
} 
Studies C and D in the online appendix provide additional support for Study 2. While Study 1 introduced multiple unclear attributes in the condition of high ambiguity, Study 2 introduced one single unclear attribute to manipulate ambiguity. Studies C-D replicate the findings of Study 2 by using a more unclear attribute than in Study 2 (Study C) or by manipulating a different product attribute (Study D). Across Study 2 and Studies C-D, we used a consumer good (a smartphone) instead of a financial product.

\section{STUDY 1}

In this study, we run an experiment on M-Turk, using a $2 \times 3$ design where we manipulate the level of ambiguity (low vs. high ambiguity) and the type of categorization process primed at the moment of evaluation (control vs. prototype-based vs. goal-based).

Participants. The experiment was conducted online, ${ }^{3}$ and participants were recruited on M-Turk. Participants were tasked to imagine that they were entrepreneurs looking to grow their venture. They were told that a bank was offering them a financial product to evaluate. Three-hundred twenty participants, receiving financial compensation, completed the study. ${ }^{4}$ Because we conducted the experiment online on M-Turk, we included an Instructional Manipulation Check (IMC) to test whether participants read the instructions (Oppenheimer et al. 2009). Fifty-four participants did not pass the attention test, and consistent with recent guidelines, we did not exclude participants who did not pass the IMC test (Berinsky et a. 2016, Vannette 2016). However, excluding these participants did not change the significance of the results reported below. Of all participants, $52 \%$ were male, and the average age of the total study population was 32.25 years [SD $=10.47$ ].

\footnotetext{
${ }^{3}$ In experimental psychology, the level of reliability of on-line experiments has proven to be similar to lab experiments (Birnbaum 2004, Dandurand et al. 2008) when experiments are characterized by "a high degree of automation of the experiment (low maintenance, limited experimenter effects" (Reips, 2002, p. 244), which is applicable to our design. The main drawback of on-line experiments is their high-risk of dropouts (Dandurand et al. 2008). To avoid such an inconvenience, we purposely designed short experiments (below 5 minutes on average for the entire experiment).

${ }^{4}$ Consistent with Burbano (2016, p. 1015), across all six studies, we excluded in total 14 participants who systematically reported extreme values (only including " 9 " or " 0 " across all question items).
} 
Priming Prototype vs Goal-Based Categorization. We created a control group, wherein participants evaluated the loan without any priming. In the prototype-based and goal-based groups, participants were asked two different sets of questions: in the prototype-based group, "Could you describe what you would expect from a traditional loan?"; in the goal-based group, "How do you think a financial product could help an entrepreneur achieve his/her strategic goals??. These manipulations ensured that the participants focused on the fit with either a prototype (i.e., a traditional loan) or a goal (i.e., a strategic goal). As per H1, we expect that the priming of a goal has a positive direct effect on the evaluation of the ambiguous loan, but not on the evaluation of the nonambiguous loan.

Level of Ambiguity. We described the loan along five attributes, consistent with the description used by Public Bank. We created two conditions: one condition of low ambiguity, and one condition of high ambiguity. Across both conditions of ambiguity, the loan description was similar in size by number of lines and word count (52 words in the low-ambiguity condition, and 50 words in the high-ambiguity condition). In the high-ambiguity condition, we increased ambiguity by connecting unrelated specificities with operators (and and or), which decreased the typicality of the offering (Barsalou, 1985). For instance, we used "fixed rate" for the loan in the low-ambiguity condition versus a "fixed or floating rate" in the high-ambiguity condition (see Appendix 1).

Dependent variable. Participants were asked to evaluate the loan on a 10 -point scale from I do not like it at all (0) to I like it very much (9). Across conditions, evaluation had a mean $=5.92(\mathrm{SD}=1.93)$. This evaluation score was the dependent variable in our analyses of variations (ANOVAs). Manipulation Check. Following evaluation, participants were asked to assess the offering on a scale ranging from non ambiguous (0) to extremely ambiguous (9). Participants found the offering significantly more ambiguous $(\mathrm{M}=5.84, \mathrm{SD}=2.01)$ in the condition of high ambiguity than in the condition of low ambiguity $(\mathrm{M}=5.29, \mathrm{SD}=2.41) \mathrm{t}(1,318)=2.22, \mathrm{p}=0.02$, two-tailed test. 
Testing the Hypotheses. First, to test whether participants' evaluations differed across conditions, we performed a 2 (low ambiguity vs. high ambiguity) $\times 3$ (control vs. prototype-based vs. goal-based categorization) ANOVA on the likability measure. The interaction effect was significant $(\mathrm{F}(2,314)=$ $3.39, \mathrm{p}=0.03)$, which provides direct evidence that evaluations differed across conditions of ambiguity and groups (control vs. prototype vs. goal).

Second, we looked at whether we could detect any differences across the control and prototype-based categorization groups. To do so, we ran a 2 (high ambiguity vs. low ambiguity) $\times 2$ (control vs. prototype-based categorization) ANOVA on the likability measure. As expected, the effects did not differ across groups, and the interaction term showed no significant effect $(\mathrm{F}(1,225)$ $=0.01, \mathrm{p}=0.91)$. This provides evidence that central tendency is the default cognitive mechanism activated by the participants when they are placed in a control condition or primed with a prototype (on-line appendix Study A supports a similar result). For the participants placed in a control group or primed with a prototype, ambiguity had a negative effect on evaluation $\left(M_{\text {High ambiguity }}=5.81, \mathrm{SD}\right.$ $=1.91$ vs $M_{\text {Low ambiguity }}=6.35, \mathrm{SD}=1.95$ vs.; $t(1,227)=-2.06, \mathrm{p}=-0.04$, two-tailed test). These participants, on average, significantly discounted the ambiguous loan offering, irrespective of the fact that they were placed in a control or prototype-based groups.

Third, we looked at whether we could detect any differences when goal-based categorization was primed. We thus ran a 2 (low ambiguity vs. high ambiguity) $\times 2$ (control vs. goal-based categorization) ANOVA on the likability measure and found a significant interaction $(F(1,208)=$ $6.56, \mathrm{p}=0.01$ ). In addition, we ran a 2 (low ambiguity vs. high ambiguity) $\times 2$ (prototype-based vs. goal-based categorization) ANOVA on the likability measure and found a significant interaction $(\mathrm{F}(1,195)=4.57, \mathrm{p}=0.03)$ as well.

Thus, it follows from the two preceding points that we should observe significant differences across groups (goal-based vs. the other two) for the high and low ambiguity conditions. Figure 1 
shows the means across conditions and groups.

\section{--Insert Figure 1 about here--}

As expected, in the high-ambiguity condition, the mean of evaluation was significantly higher in the goal-based group than in the control group $\left(M_{\text {Goal }}=6.60, \mathrm{SD}=1.63\right.$ vs. $M_{\text {Control }}=5.90, \mathrm{SD}=$ 1.73 vs.; $t(1,103)=2.04, \mathrm{p}=0.04$, two-tailed test $)$ and the prototype-based group $\left(M_{\mathrm{Goal}}=6.60, \mathrm{SD}\right.$ $=1.63$ vs. $M_{\text {prototype }}=5.72, \mathrm{SD}=2.11 ; t(1,93)=2.21, \mathrm{p}=0.03$, two-tailed test), providing direct support for H1. Furthermore, in the low-ambiguity condition, the mean of evaluation was significantly lower in the goal-based group than in the control group $\left(M_{\mathrm{Goal}}=5.92, \mathrm{SD}=1.74\right.$ vs. $M_{\text {Control }}=6.47, \mathrm{SD}=1.79 ; t(1,105)=-1.60, \mathrm{p}=0.1$, two-tailed test $)$ and in the prototype-based $\operatorname{group}\left(M_{\mathrm{Goal}}=5.92, \mathrm{SD}=1.74\right.$ vs. $M_{\text {Prototype }}=6.22, \mathrm{SD}=2.10 ; t(1,102)=-0.79, \mathrm{p}=0.43$, two-tailed test), although this difference was not significant. Thus, when evaluating a non-ambiguous product offering, priming a goal is marginally detrimental to evaluation, as a more automatic categorization process based on the cognitive mechanism of central tendency is preferred.

In addition, we ran a set of tests to exclude the possibility that the perception of complexity drove our results. Participants were asked to rate their ability to understand the offering on a scale of easy (0) to difficult (9) to understand and whether they found the offering complex, with possible answers ranging from absolutely not complex (0) to extremely complex (9). We were unable to detect any variation across conditions of ambiguity or across groups (i.e. control vs. prototype vs. goal) in terms of (i) the perception of whether the offering was easy or difficult to understand $(F(2,314)=0.93, p$ $=0.4$ ) or (ii) the perception of complexity $(\mathrm{F}(2,314)=1.06, \mathrm{p}=0.35)$. In addition, we compared the means of these two variables across our ambiguity conditions, and we were unable to detect a difference at $\mathrm{p}<0.1$ in both cases. Finally, we ensured that the priming of a goal did not trigger a substantially distinct thinking process (Kahneman and Frederick 2005). To do so, we measured, in 
seconds, the time that participants spent reading about the loan across conditions following the priming on a goal and the priming on a prototype and we were unable to detect any significant different across conditions $(\mathrm{F}(2,314)=1.28, \mathrm{p}=0.28)$.

Taken together, these results provide strong support for our main hypothesis: activation of goal-based categorization leads to a positive evaluation of ambiguous products.

\section{Replicating the results with a population of real-world entrepreneurs}

Study 1's main finding showed that the priming of a goal had a positive direct effect on the evaluation of an ambiguous product (in our case, a loan), compared with the priming of prototypebased categorization or when participants are placed in a control condition. However, because Public Bank's product was targeted to an audience of business professionals and entrepreneurs, we run a similar experiment with entrepreneurs using the same financial product, thereby improving the ecological validity of our results. The on-line appendix (Study B) presents the experiment with realworld entrepreneurs in full. Note that the research protocol, notably, the low/high ambiguity and the manipulations of prototype-based/ goal-based categorization remained unchanged. Overall, Study B provides similar results with Study 1: entrepreneurs significantly discounted the ambiguous loan offering when primed with a prototype and the evaluation of the ambiguous loan was higher when goal-based categorization was primed (compared to the prototype condition).

\section{STUDY 2}

Previous studies (Study 1 as well as Studies A and B reported in the on-line appendix) provide evidence that priming goal-based categorization has a positive effect on the evaluation of an ambiguous product. However, while these conclusions stand, we cannot totally exclude the possibility that our results are applicable only to certain products. Furthermore, we need to provide evidence, as hypothesized in $\mathrm{H} 2$, that the presence of a goal in mind coincides with the activation of conceptual combination, which rewards the effort put in the task of evaluating unclear attributes in 
relation to a pursued goal, thus translating into an increase in the perceived utility of unclear product attributes. To address these points, we conducted a new experiment on M-Turk.

First, participants evaluated a consumer good, which increased the generalizability of our findings. Second, we measured, for each attribute, whether or not participants found a given product attribute to be useful. By so doing, we can measure whether goal-based categorization does increase the perceived utility of an unclear product attribute (vs. clear product attributes), which, in turn, impacts positively the evaluation of an ambiguous product offering.

Participants. Participants, who were recruited on M-Turk, were asked to imagine that they were buying a new phone. Participants were randomly assigned to a 2 (high/low ambiguity product) $\times 3$ (control/ prototype-based/goal-based groups) study design. Three-hundred and seventeen participants, receiving financial compensation, completed the study. Because the experiment was conducted online on M-Turk, we included an IMC test (Oppenheimer et al. 2009). Seventeen participants failed this attention test; and, consistent with recent guidelines, we did not exclude participants who did not pass the IMC test (Berinsky et al. 2016, Vannette 2016). However, their exclusion did not change the significance of the results reported below. Of all participants, 49\% were male, and the average age was 37.16 years [ $\mathrm{SD}=12.08]$.

Priming Prototype vs Goal-Based Categorization. In this experiment, we primed prototype vs. goal-based categorization immediately before participants' evaluation, using priming techniques consistent with those used in Study 1. In the prototype-based and goal-based groups, participants were asked two different questions: in the prototype-based group, "Could you describe what you would expect from a traditional mobile phone?"; in the goal-based group, "How do you think a mobile device could help you watching movies on the go?" These manipulations ensured that the participants focused on the fit with either a prototype (i.e., a traditional mobile phone) or a goal (i.e., watching movies on the go). 
Level of Ambiguity. Consistent with Study 1, each product offering across the low- and highambiguity conditions presented five product attributes. Because we hypothesized that the introduction of one unclear attribute can generate ambiguity for the evaluator, in this study, we introduced only one unclear attribute, holding constant other product attributes presented across conditions (see Appendix 2). We hypothesized that the unclear attribute should be at least minimally relevant to the goal at hand for the mechanism to operate. Arguably, priming the ad hoc goal of watching movies on the go should not have any influence if we'd have manipulated, say, the processor's characteristics. This is why we decided to manipulate the capacity to use data. As such, participants in the low-ambiguity condition read that the product offering has " $4 \mathrm{G}$ surfing speed $(6 \mathrm{GHz})$, perfect for optimal web browsing", whereas participants in the high-ambiguity condition read that the product offering has " $4 \mathrm{G}$ surfing speed with floating capacity of plus or minus 3 to $9 \mathrm{GHz}$ ". Taken together, introducing only one unclear attribute has two advantages: we can show that (1) introducing a single unclear attribute generates ambiguity for the evaluator, and (2) priming goal-based categorization substantially increases the perceived utility of this specific product attribute (but not the perceived utility of the clear attributes), impacting, in turn, the evaluation of the ambiguous product offering.

\section{Dependent variable:}

As per Study 1, we asked participants to evaluate the offering on a 10-point scale from I do not like it at all (0) to I like it very much (9). Following Gibbert and Mazursky (2009, p. 655), to go beyond likability, we measure also the perceived market success of the offering by asking participants whether they thought the offering would be successful, with possible answers ranging from not at all (0) to absolutely (9). Our dependent variable, evaluation, is thus a combined measure of likability and perceived market success. ${ }^{5}$ As highly correlated, evaluation is the average of the likability score and the

\footnotetext{
${ }^{5}$ We run a set of analyses on each measure, and found highly consistent results with the ones presented here.
} 
perceived market success score $(\mathrm{r}=0.80, \mathrm{p}<0.01)$, has a mean $=6.83(\mathrm{SD}=1.56)$ and is the dependent variable used in Study 2.

\section{Perceived utility of attributes}

Following evaluation, we asked participants to evaluate the utility of each attribute presented in the offering. To do so, we asked "How would you rate the utility of the following feature?" on a 10point scale, from not useful at all (0) to very useful (9). To test our hypothesis (H2) that the perceived utility of the unclear attribute (1) increases under goal-based categorization (relative to a control condition or to prototype-based categorization) and (2) positively impacts the evaluation of an ambiguous product offering, we used the perceived utility of the unclear product attribute as a mediator variable. In addition, we created another score, which averages the perceived utility of the remaining four clear attributes that were held constant across conditions of ambiguity, to test whether priming a goal impacted the evaluation of clear attributes as well. We thus labelled perceived utility of the unclear attribute, the perceived utility of the attribute that we manipulated, and this score had a mean $=6.74(\mathrm{SD}=2.11)$ across groups and conditions. In addition, the perceived utility of clear attributes — the average of the four clear attributes that remain identical across ambiguity conditions; see appendix 2 - had a mean $=7.16(\mathrm{SD}=1.46)$ across groups and conditions. Overall, our prediction is that only the perceived utility of the unclear attribute differs across groups (control vs. prototype-based vs. goal-based) but not the perceived utility of the clear attributes.

Manipulation Checks. Before running our experiment, we ran a pretest on M-Turk with 95 participants $-40 \%$ participants were male, and the average age was 39.21 years [SD $=10.89$ ] investigate whether participants placed in the high- ambiguity condition perceived the offering as being more ambiguous and whether their ability to associate the unclear attribute with the smartphone product category was impaired by our ambiguity manipulation. Using the same score presented in Study 1 to capture perceived ambiguity, we found that participants perceived the 
offering as a whole to be significantly more ambiguous $(\mathrm{M}=4.76, \mathrm{SD}=2.09)$ in the high-ambiguity condition than in the low-ambiguity condition $(\mathrm{M}=3.71$, $\mathrm{SD}=2.67) \mathrm{t}(1,91)=2.12, \mathrm{p}=0.03$, twotailed test). In addition, depending on the ambiguity condition, participants were asked whether they would, on a 10-point scale, "immediately associate” the clear attribute (“4G surfing speed (6GHz), perfect for optimal web browsing") or the unclear attribute (" $4 \mathrm{G}$ surfing speed with floating capacity of plus or minus 3 to $9 \mathrm{GHz}$ ”) with the smartphone product category. Overall, as expected, participants were more likely to associate the clear " $4 \mathrm{G}$ surfing speed" product attribute with the smartphone product category than its version; $(M=7.14, \mathrm{SD}=1.69)$ for clear product attribute compared to $(\mathrm{M}=6.30, \mathrm{SD}=2.29), \mathrm{t}(1,95)=2.03, \mathrm{p}=0.04$, two-tailed test $)$ for the unclear attribute. Testing the Hypotheses. First, to test whether participants' evaluation differed across conditions and groups, we performed a 2 (low ambiguity vs. high ambiguity) $\times 3$ (control vs. prototype-based vs. goal-based categorization) ANOVA on the evaluation score measure. The interaction effect was significant $(\mathrm{F}(2,311)=2.76, \mathrm{p}=0.06)$, which provides direct evidence that our evaluation score differed across conditions of ambiguity and groups (control vs. prototype-based vs. goal-based). Figure 2 shows the means of this evaluation score across the six conditions.

\section{--Insert Figure 2 about here-}

Second, we checked whether we could detect any differences across the control and prototype-based categorization groups. We ran a 2 (low ambiguity vs. high ambiguity) $\times 2$ (control vs. prototype-based categorization) ANOVA on our evaluation score. As expected, the effects did not differ across conditions and groups, and the interaction term had no significant effect $(F(1,203)=$ 0.01, $\mathrm{p}=0.91$ ). Consistent with Study 1 (and Studies A and B reported in the on-line appendix), ambiguity had a negative effect on evaluation $\left(M_{\text {High ambiguity }}=6.53, \mathrm{SD}=1.80\right.$ vs. $M_{\text {Low ambiguity }}=$ $6.97, \mathrm{SD}=1.37 ; t(1,205)=-2.02, \mathrm{p}=0.04$, two-tailed test) when participants were placed in a control group or primed with a prototype, which shows that these participants, on average, 
significantly discounted the ambiguous mobile phone.

Third, as per Study 1, we run a set of two different ANOVAs on our evaluation score. We ran a 2 (low ambiguity vs. high ambiguity) $\times 2$ (control vs. goal-based categorization) ANOVA on evaluation and found a significant interaction $(\mathrm{F}(1,210)=4.32, \mathrm{p}=0.04)$. In addition, we ran a 2 (high ambiguity vs. low ambiguity) $\times 2$ (prototype-based vs. goal-based categorization) ANOVA on evaluation and found a significant interaction $(\mathrm{F}(1,209)=4.05, \mathrm{p}=0.05)$ as well, providing evidence that evaluation substantially differed across groups and conditions of ambiguity.

As expected, in the high-ambiguity condition, the mean of evaluation was significantly higher in the goal-based group than in the control group $\left(M_{\text {Goal }}=7.15, \mathrm{SD}=1.32\right.$ vs. $M_{\text {Control }}=6.43, \mathrm{SD}=$ $1.90 ; t(1,103)=2.31, \mathrm{p}=0.02$, two-tailed test $)$ and the prototype-based group $\left(M_{\mathrm{Goal}}=7.15, \mathrm{SD}=\right.$ 1.32 vs. $M_{\text {prototype }}=6.62, \mathrm{SD}=1.70 . ;(1,104)=1.82, \mathrm{p}=0.07$, two-tailed test), providing direct support for H1. Furthermore, in the low-ambiguity condition, the mean of evaluation was lower in the goal-based group than in the control group $\left(M_{\text {Goal }}=6.75, \mathrm{SD}=1.64\right.$ vs. $M_{\text {Control }}=6.90, \mathrm{SD}=$ $1.35 ; t(1,107)=-.54, \mathrm{p}=0.59$, two-tailed test $)$ and the prototype-based group $\left(M_{\mathrm{Goal}}=6.75, \mathrm{SD}=\right.$ 1.64 vs. $M_{\text {Prototype }}=7.04, \mathrm{SD}=1.39 ; t(1,105)=-1.02, \mathrm{p}=0.31$, two-tailed test). Although these results did not reach significance, it is clear indication of the contrasted effects of goal-based categorization depending on the level of ambiguity.

Consistent with previous studies, we ran a series of ANOVAs on (i) the perception of complexity, (ii) the time spent on the manipulation question, and (iii) the time spent reading the product offering. The interactions remained insignificant at $\mathrm{p}<0.05$, as per previous studies.

\section{Goal-Based Categorization, Unclear Attribute's Perceived Utility and Evaluation.}

We next investigated whether the perceived utility of the unclear attribute differs across groups (control vs. prototype-based vs. goal-based) and conditions of ambiguity. We ran a 2 (low ambiguity vs. high ambiguity) $\times 3$ (control vs. prototype-based categorization vs. goal-based categorization) 
ANOVA on the perceived utility of the unclear attribute, which yielded a significant interaction $(\mathrm{F}(2$, $311)=3.15, \mathrm{p}=0.04)$. This result indicates that the perceived utility of the unclear attribute differs as a function of the ambiguity condition (low vs. high) and the categorization process primed (control or prototype-based vs. goal-based categorization). Next, we investigated whether the perceived utility of the clear attribute (" $4 \mathrm{G}$ surfing speed $(6 \mathrm{GHz})$, perfect for optimal web browsing") differs across groups. We thus run a one-way ANOVA (control or prototype-based vs. goal-based categorization) on the perceived utility of this clear attribute, and the main effect was not significant $(F(2,160)=.37, \mathrm{p}=0.69)$. This test provides evidence that the perceived utility of the "4G surfing speed" clear attribute did not differ across groups, that is irrespective of the fact that participants were placed in a control group or primed with prototype-based or goal-based categorization, the perceived utility of this attribute did not vary significantly. By contrast, we ran a one-way ANOVA (control or prototype-based vs. goal-based categorization) on the perceived utility of the unclear attribute ("4G surfing speed with floating capacity of plus or minus 3 to $9 \mathrm{GHz}$ "), and the main effect was significant $(F(2,151)=3.11, \mathrm{p}=0.05)$. The mean of the perceived utility of the unclear attribute was significantly higher in the goal-based group than in the control group $\left(M_{\mathrm{Goal}}=\right.$ $6.56, \mathrm{SD}=1.94$ vs. $M_{\text {Control }}=5.48, \mathrm{SD}=2.51 ; t(1,103)=2.49, \mathrm{p}=0.01$, two-tailed test $)$ or the prototype-based group $\left(M_{\text {Goal }}=6.56, \mathrm{SD}=1.94\right.$ vs. $M_{\text {prototype }}=5.84, \mathrm{SD}=2.41 ; t(1,104)=1.72, \mathrm{p}$ $=0.09$, two-tailed test). As such, goal-based categorization impacts the perceived utility of the unclear attribute, but not the clear "4G surfing speed" attribute.

In addition, we tested whether the average of the remaining four clear attributes that were identical across ambiguity conditions differed or not across groups or ambiguity conditions. To do so, we ran a 2 (low ambiguity vs. high ambiguity) $\times 3$ (control vs. prototype-based categorization vs. goal-based categorization) ANOVA on the perceived utility of the clear attributes, which yielded a non-significant interaction $(\mathrm{F}(2,311)=1.56, \mathrm{p}=0.21)$. Note that in both low and high ambiguity 
conditions, this score did not differ across groups, yielding a non-significant one-way ANOVA at $\mathrm{p}<.1$ in both cases. Taken together, these tests showed that goal-based categorization only impacts the perceived utility of the unclear attribute, and not the perceived utility of the clear attributes.

We next tested whether the positive effect of goal-based categorization on the evaluation of an ambiguous product offering was due to an increase in the perceived utility of the unclear product's attribute. Consequently, to test $\mathrm{H} 2$, we ran a mediation analysis to determine whether the perceived utility of the unclear attribute mediated the effect of goal-based categorization on evaluation. To conduct the mediation analyses, we used the PROCESS macro in SPSS developed by Hayes $(2015,2017)$. We ran an analysis that contrasted the goal-based group in two ways: first with "control group" and then with "prototype-based group." Figure 3 displays the results, with coefficients in gray corresponding to the "control" contrast, while the coefficient in blue corresponds to the "prototype" contrast. As shown in Figure 3, goal-based categorization, compared to either group, has a positive effect on the perceived utility of the unclear attribute (path a: beta $_{1}=$ 0.541, $\mathrm{p}=0.01, \mathrm{CI}[0.11 ; 0.97] ;$ beta $\left._{2}=0.724, \mathrm{p}=0.09, \mathrm{CI}[-0.11 ; 1.56]\right)$ and, perceived utility has, in turn, a positive effect on the evaluation of the ambiguous offering (path $b$; beta ${ }_{1}=0.340, p=0.000$, CI $[0.214 ; 0.467] ;$ beta $_{2}=0.145, p=0.03$, CI $\left.[0.01 ; 0.28]\right)$. Finally, a bias-corrected bootstrap based on 10,000 bootstrap samples showed that the interval for the indirect effect was entirely above zero when comparing goal-based categorization to a control group (0.026-0.423) — and includes 0 when comparing goal-based categorization to a prototype-based group (-0.015-0.319). Notably, the mediation in both contrast analyses was strong enough to cancel out the direct effect of goal-based categorization on evaluation that we found earlier (see path c in Figure 3).

--Insert Figure 3 about bere--

\section{Replicating results}

First, we replicated the findings presented above using a different dependent variable: the evaluation 
score framed in a third-order way (Correll et al. 2017). As such, in Study 2, we asked participants whether they thought other people would like this product on a 10-point scale from not at all (0) to absolutely (9) and whether they thought other people would think that this product is likely to be successful, with possible answers ranging from not at all (0) to absolutely (9). Our third-order evaluation score is the average of the two variables and has a mean $=7.09(\mathrm{SD}=1.51)$. Then, we ran the $2 \times 3$ ANOVA on our third-order evaluation score. Consistent with the findings reported in Study 2, the interaction effect was significant $(\mathrm{F}(2,311)=3.50, \mathrm{p}=0.03)$, which shows that this score also substantially differed across conditions and groups. We followed up with comparison of means and we found consistent and similar results with the ones presented in the result section of Study 2 .

Second, we replicated the results above and ran a new study which increased the level of ambiguity of the product: we increased how much unclear the " $4 \mathrm{G}$ surfing speed attribute" is. In addition, we ran another study where we manipulated a different product attribute (the "display" vs. "4G surfing speed") to ensure that the specific attribute that we chose did not drive the effects. We present both studies in full in the on-line appendix (see Studies C and D, respectively).

In addition, our theory implies that the assessment of individual product attributes leads to a higher valuation of ambiguous products when goal-based categorization is primed because participants associate some utility of the unclear attribute with a goal they pursue. If this theory is true, those participants with greater aptitude at abstracting and greater knowledge of the categories will be more likely to able to see potential value in unclear attributes with respect to the goal to be achieved (Barsalou 1991, Medin et al. 1997, Lo and Kennedy 2015, Lynch et al. 2000, Johnson and Mervis 1997, Boulongne et al. 2019). To do so, we also tested whether knowledgeability impacted the perceived utility of the unclear attribute.

Overall, these two additional studies show similar results to the ones presented in Study 2: goal-based categorization does increase the evaluation of the ambiguous offering, and this effect is 
mediated by the perceived utility of the unclear attribute. These results are consistent when (1) we increase the level of ambiguity of the offering (see Study C) or (2) we use a different product attribute (the display, see Study D). Notably, Study D provides evidence that priming goal-based categorization leads to negatively impact the evaluation of the non-ambiguous mobile phone, which directly echoes the negative effect, although non-significant, found in the context of Study 2. In addition, we found evidence in these two additional studies that knowledgeability positively moderates the mediation via the perceived utility of the unclear attribute as expert audience members see a greater utility in the unclear attribute when goal-based categorization is primed.

\section{DISCUSSION}

Our study examined the conditions that influence how audience members evaluate an ambiguous product offering. The implications of understanding how audience members make sense of ambiguity loom large for research on firm strategy, market categories, and audiences' ascription of value to innovation. While existing research defines the factors that mitigate the negative effects of ambiguity on evaluation $^{6}$, no study to date tested why priming goal-based categorization improves the evaluation of ambiguous offerings in markets.

Across multiple studies, we show why and how goal-based categorization positively impacts the evaluation of ambiguous offerings. First, we confirm that when evaluators rely on central tendency — when they are primed on prototype-based categorization or placed in a control groupthey tend to discount an ambiguous offering. More importantly, we find that goal-based categorization has a direct positive effect on the evaluation of an ambiguous product (in our studies, a loan and a smartphone). When audiences are primed on goal-based categorization, they switch off

\footnotetext{
${ }^{6}$ See Durand and Boulongne (2017): For the former, category contrast (Negro et al. 2010, Kovács and Hannan 2015), category leniency (Pontikes 2012), status (Sharkey 2014, Montauti and Wezel 2016), and organizational age (Carnabuci et al. 2015); for the latter, audiences' goal sophistication and theories of value (Paolella and Durand 2016), and producers' labelling strategies (Bowers 2015, Granqvist et al. 2013, Wry et al. 2014).
} 
central tendency, see a greater utility in unclear attributes, and more favorably evaluate the ambiguous product in relation to a goal. Finally, our studies show some evidence that goal-based categorization reduces the evaluation of non-ambiguous products because (i) similarity check with a prototype is preferred when evaluating non-ambiguous products and (ii) priming a goal is rewarding for the evaluator when the task at hand is cognitively demanding (Labroo and Kim, 2009).

First, our results refocus our attention on the effects of ambiguity on evaluation, and away from the specific case of category spanning: whereas past research has thoroughly investigated the effects of category spanning (Hsu 2006, Hsu et al. 2009) and category ambiguity on evaluation (Fleischer, 2009; Pontikes, 2012), scholars have lost sight that ambiguity is created when an audience member cannot relate a product attribute to a product category. Therefore, ambiguity can also be generated via the introduction of one or multiple unclear attribute(s) from a given category, without spanning. While it is certainly true that when products and organizations borrow attributes from competing and distinct categories, they create ambiguity for evaluators, it is equally true that the introduction of a single unclear attribute in a product definition hinders audience members' capacity to rely on central tendency, thus ultimately leading to discount such an ambiguous offering when an ad hoc goal is not readily primed (Barroso et al. 2016, Durand et al. 2007). While most of prior studies could not estimate what an offering's evaluation would be had audiences been primed with a different categorization process, experimental designs allow us to do this. All in all, our work both complement and redirect existing research on categories by stressing the importance of looking at attributes in isolation together with the priming of specific categorization processes and the activation of distinct cognitive mechanisms.

Second, our results show that the categorization context matters (Durand et al, 2017). Audiences do not systematically penalize ambiguity in markets: priming goal-based categorization increases the evaluation of ambiguous offerings and experts are more capable at activating 
conceptual combination (Study C and D, online). However, we find evidence also across our studies that in condition of low ambiguity, priming goal-based categorization leads to lower assessment of offerings (See Study 1 and Study D in the on-line appendix for more details). Overall, our paper provides evidence that we should refrain from inferring or imposing all-encompassing properties on market actors at the audience level but instead strive to determine which cognitive mechanism (central tendency or conceptual combination) each audience member activates in a given situation when facing a producer's offering.

Third, our findings have implications for firm strategy and directly speak to an unresolved strategic issue: should firms strive to be the same as or different from their rivals (Deephouse 1999)? Zhao et al.'s (2017) strategic “optimal distinctiveness” is and remains a fundamental research puzzle. The debate remains open, in part because all prior studies have inferred-but none has testedcognitive mechanisms on the audience side (e.g., customers, raters, or investors). However, it is impractical to simply assess a distance between a firm and the typical firm, and associate that distance with an outcome (e.g., performance in Deephouse 1999, or reputation gain in other studies -e.g. Durand et al. 2007) without first determining who assessed the distance and how. The missing lever to understand when it is better to be similar or dissimilar from the typical firm is therefore the understanding of the conditions under which an audience member activates a given cognitive mechanism. As Kahl and Grodal (2016, p. 149) pointed out - in their example of IBM's strategy of making the then new computer products seem familiar while Remington Rand strove to make them seem novel_ “firms have opportunities to influence strategically customers' interpretations of the technology in ways that favor their offerings." As such, when audience members evaluate a firm's offerings, they respond to prompts and activate various cognitive mechanisms (Zhao et al. 2017). We have shown the conditions under which the priming of goal-based categorization, by positively impacting the evaluation of ambiguous product offerings, helps differentiators to benefit from 
positive evaluations, thereby building up the cognitive underpinnings that lead them to achieve greater performance.

These findings can help managers to deal with ambiguity when they manage their existing product portfolios, introduce novel offerings into markets, or create new product categories (Bingham and Kahl 2013, Kahl and Grodal 2016). Other studies have identified the role of an audience's cognitive interpretations of a firm's offerings as being instrumental in driving competition (see Barnett and McKendrick 2004), and more recently, Anthony et al (2016, p. 178) stressed that “cognitive interpretations, not technical features, shape competition.” As such, our approach calls for a better consideration of how firms generate the conditions surrounding an audience's selection of their offerings at a cognitive level—i.e., within a category system. Taken together, firms must strategically manage product differentiation, the mix between well-established (i.e., clear) and nonestablished (i.e., unclear) attributes, and the cognitive mechanisms that audiences activate to process producers' offerings. This study complements more traditional approaches of competition (Cattani et al, 2017) and points to a fundamentally better specification of individual responses to ambiguity and cognitive priming strategies. When proposing a product that contains unclear attributes, firms are better off triggering goal-based categorization in their audiences by stressing a goal, an ideal, or a mission that will be made accessible through their offerings. The results of Study 1 (as well as Studies A and B in the on-line appendix) were presented to Public Bank's executive committee (January 2017). Based on this, Public Bank decided to revise its online and offline commercialization strategies for the loan used as reference in these studies.

Before concluding, we stress three limitations of this work. First, we assigned goals that remain in the ambit of the entity under consideration. We assumed—but did not test— that our mechanism would not operate when the unclear attributes primed are completely irrelevant to the goal at hand. This deserves further confirmation. Second, our main comparison sets were "control" 
(i.e., no priming) and "prototype-based." Recent research indicates that other baselines, such as exemplars, are explanatory of categorization (Zhao et al. 2018). Likewise, producers can prime other categorization processes, such as analogies (Bingham and Kahl, 2013, Etzion and Ferraro, 2010). Additional studies are needed to compare and contrast which condition is amenable to higher or lower valuations of ambiguous products and organizations. Third, we did not provide detailed information about the producers in any of our studies. It is likely that the producer's status or its competence, among other factors, could accentuate or temper the observed relationships, and thus should be accounted for in future studies (e.g., Montauti and Wezel 2016). In the same line of thought, third parties influence how well the producers' intended strategies reach audiences and activate the expected evaluative cognitive mechanism.

To conclude, this work provides evidence that when activated, goal-based categorization leads to a positive evaluation of ambiguous offerings (and not when it comes to the evaluation of non-ambiguous products). Thus, this work invites future research to account for the actual cognitive mechanisms activated by each audience member when they categorize and evaluate organizations and their products. It calls also for understanding better why superior value accrues to organizations that offer ambiguous products by intendedly priming potential buyers' different categorization processes. 


\section{REFERENCES}

Anthony C, Nelson AJ and Tripsas M (2016) Who Are You?...I Really Wanna Know": Product Meaning and Competitive Positioning in the Nascent Synthesizer Industry. Strat. Science. 1:163-183.

Barnett WP, and McKendrick D (2004) Why Are Some Organizations More Competitive Than Others? Evidence from a Changing Global Market. Adm. Science Quar. 49, 535-571

Barroso A, Giarratana M, Reis S and Sorenson O (2016) Crowding, satiation, and saturation: The Days of television series' lives, Strat. Management Jour., 37: 565-585.

Barsalou, L.W (1983) Ad hoc categories. Memory \& Cognition, 11, 211-227.

Barsalou LW (1985) Ideals, central tendency, and frequency of instantiation as determinants of graded structure in categories. J. of Exp. Psych.: Learning, Memory, and Cognition, 11, 629-654.

Barsalou, LW (1991) Deriving categories to achieve goals. In G. H. Bower (Ed.), The psychology of learning and motivation: Advances in research and theory, Vol. 27: 1-64. San Diego, CA: Academic Press.

Benner M and Tripsas M (2012) The influence of prior industry affiliation on framing in nascent industries: the evolution of digital cameras. Strategic Management J. 33, 277-302

Berinsky AJ, Margolis MF and Sances MW (2016) Can We Turn Shirkers Into Workers? J. of Expe. Social Psych. 66: 20-28

Bingham CB and Kahl SJ (2013) The process of schema emergence: Assimilation, deconstruction, unitization and the plurality of analogies. Acad. of Management J. 56:14-34

Birnbaum MH (2004). Methodological and ethical issues in conducting social psychology research via the Internet. In Handbook of Methods in Social Psychology, ed. C. Sansone, C.C. Morf, A.T. Panter. 359-82. Thousand Oaks, CA: Sage

Boulongne, R., Cudennec, A., \& Durand, R. (2019). When do market intermediaries sanction categorical deviation? The role of expertise, identity, and competition. Res. In the Soc. of Organizations. In P. Haack, J. Sieweke, \& L. Wessel (Eds.), Microfoundations of institutions (Vol. 65A). Bingley: Emerald.

Bowers A (2015) Relative comparison and category membership: The case of equity analysts. Org. Science 26(2):571-583.

Burbano VC (2016) Social Responsibility Messages and Worker Wage Requirements: Field Experimental Evidence from Online Labor Marketplaces. Org. Science. 27(4): 1010 - 1028

Cattani G, Porac J, and Thomas H (2017) Categories and Competition. Strategic Management J., 38(1): 64-92.

Carnabuci G, Operti E and Kovács B (2015) Categorical imperative and structural reproduction: Insights from the global semiconductor industry. Org. Science 26(6): 1734-1751.

Chrysikou, E. (2006). When shoes become hammers: Goal-derived categorization training enhances problem-solving performance. J. of Experimental Psychology: Learning, Memory, and Cognition, 32, 935-942.

Cusumano MA, Kahl SJ and Suarez FF (2015) Services, Industry Evolution, and the Competitive Strategies of Product Firms. Strategic Management J. 36(4):559 575.

Correll, S.J., Ridgeway, C.L., Zuckerman, E.W., Jank, S., Jordan-Bloch, S. and Nakagawa, S., 2017. It's the conventional thought that counts: How third-order inference produces status advantage. Am. Soc. Rev., 82(2), pp.297-327.

Dandurand F, Shultz TR and Onishi KH (2008) Comparing online and lab methods in a problem-solving experiment. Behavior Res. Methods. 40 (2), 428-434

Deephouse DL (1999) To be Different, or to be the same? It's a Question (and theory) of Strategic balance. Strategic Management J. 20: 147-166.

Durand, R., \& Boulongne, R. (2017). Advancing category research: Theoretical mapping and under-researched areas. The Sage handbook of organizational institutionalism, 647-668.

Durand, R., Granqvist, N., \& Tyllström, A. (2017). From Categories to Categorization: A Social Perspective on Market Categorization', From Categories to Categorization: Studies in Sociology, Organizations and Strategy at the Crossroads (Research in the Sociology of Organizations) Volume 51:3-30.

Durand R and Paolella L (2013) Category stretching: Reorienting research on categories in strategy, entrepreneurship, and organization theory. Jour. of Management Stud. 50: 1100-1123.

Durand R, Rao H and Monin P (2007) Code and conduct in French cuisine: impact of code changes on external evaluations. Strategic Management J. 28, 455-72.

Etzion D and Ferraro F (2010) The Role of Analogy in the Institutionalization of Sustainability Reporting. Org. Science. 21(5): 1092-110

Fiske ST and Pavelchak MT (1986) Category-based versus piecemeal-based affective responses: Developments in schema-triggered affect.' In R. M. Sorrentino and E. T. Higgins (eds. \}. Handbook of Motivation and Cognition: Foundations of Social Behavior: 167-203. New York: Guilford.

Fiske ST and Neuberg SL (1990) A continuum of impression formation, from category-based to individuating processes: 
Influences of information and motivation on attention and interpretation'. In M. P. Zanna (Ed.), Advances in experimental social psychology (Vol. 23 (pp. 1-74). New York: Academic Press.

Fleischer, A. (2009). 'Ambiguity and the equity of rating systems: United States brokerage firms, 1995-2000'. Ad. Science Quar., 54, 555-74.

Gibbert M and Mazursky D 2007 A recipe for creating new products, Wall Street Journal

Gibbert, M., \& Mazursky, D. 2009. How successful would a phone-pillow be: Using dual process theory to predict the success of hybrids involving dissimilar products. Journal of Consumer Psychology, 19: 652-660

Gibbert, M., Hampton, J. A., Estes, Z., \& Mazursky, D. (2012). The curious case of the refrigerator- TV: similarity and hybridization. Cognitive Science, 36, 992-1018.

Goldberg, A., Hannan \&M., Kovács., B. (2016). What Does It Mean to Span Cultural Boundaries? American Sociological Review, 81(2): 215-241.

Granqvist N, Grodal S and Woolley JL (2013) Hedging your bets: Explaining executives' market labeling strategies in nanotechnology'. Org. Science. 24: 395-413

Gregan-Paxton J, Hoeffler S and Zhao M (2005) When Categorization Is Ambiguous: Factors that Facilitate the Use of a Multiple Category Inference Strategy. Jour. of Consumer Psych. 15: 127-140

Grégoire DA, Barr PS and Shepherd DA (2010) Cognitive Processes of Opportunity Recognition: The Role of Structural Alignment. Org. Science 21(2):413-431

Grodal S, Gotsopoulos A and Suarez FS (2015) The Co-Evolution of Categories and Designs during Industry Emergence. Acad. of Management Rev., 40(3); 423-445.

Hannan MT, Pólos L, Carroll GR (2007) Logics of Organization Theory: Audiences, Codes, and Ecologies (Princeton University Press, Princeton, NJ).

Harrison GW and List JA (2004) Field experiments. Jour. of Econ. Lit. 42: 1009-1055

Hayes AF (2015) An index and test of linear moderated mediation. Multivariae Behavioral Res. 50, 1-22

Hayes AF (2017) Introduction to mediation, moderation, and conditional process analysis: A regression-based approach. New York, NY: The Guilford Press. Second Edition.

Hsu G (2006) Jacks of all trades and masters of none: Audiences' reactions to spanning genres in feature film production. Adm. Science Quar. 51:420-450.

Hsu G, Koçak O and Hannan MT (2009) Multiple category memberships in markets: An integrative theory and two empirical tests. Am. Sociological Rev. 74: 150-169.

Jensen M and Kim BK (2014) Great, Madama Butterfly Again! How Robust Market Identity Shapes Opera Repertoires. Org. Science 25(1):109-126

Johnson KE and Mervis CB (1997) Effects of varying levels of expertise on the basic level of categorization. Jour. of Exper. Psych.: General, 126(3), 248-277.

Kahl SJ (2015) Product Conceptual Systems: Toward a Cognitive Processing Model, in Giovanni Gavetti, William Ocasio (ed.) Cognition and Strategy (Advances in Strategic Management, Volume 32) Emerald Group Publishing Limited, pp.119- 146.

Kahl SJ and Grodal S (2016) Discursive strategies and radical technological change: Multilevel discourse analysis of the early computer (1947-1958). Strategic Management J. 37(1):149-166.

Kahneman D and Frederick S (2005) A model of heuristic judgment. In K. J. Holyoak \& R. G. Morrison (Eds.), The Cambridge handbook of thinking and reasoning (267-293). New York, NY, US: Cambridge University Press.

Klingebiel R and Joseph J (2016) Entry timing and innovation strategy in feature phones. Strategic Management J. 37(6):1002-1020

Kopetz C and Kruglanski AW (2008) Effects of accessibility and subjective relevance on the use of piecemeal and category information in impression formation. Pers. and Social Psych. Bulletin, 34, 692-705

Kovács B and Hannan MT (2015) The Space of Categories and the Consequences of Category Spanning. Socio. Science. 2: 252-286

Labroo., A, A.; Kim,S., "instrumentality; heuristic: Why metacognitive difficulty is desirable during goal pursuit" Psychological Science. 2009;20(1):127-134.

Leung MD and Sharkey AJ (2014) Out of sight, out of mind? Evidence of perceptual factors in the multiple- category discount. Org. Science. 25: 171-184

Lo YC and Kennedy MT (2015) Approval in Nanotechnology Patents: Micro and Macro Factors that Affect Reactions to Category Blending. Org. Science 26: 119 - 139

Lynch EB, Coley JD and Medin DL (2000) Tall is typical: Central tendency, ideal dimensions and graded category structure among tree experts and novices. Mem. and Cog. 28(1), 41-50. 
Medin, D. L., Lynch, E. B., Coley, J. D., \& Atran, S. 1997. Categorization and reasoning among tree experts: Do all roads lead to Rome? Cog. Psych., 32, 49-96.

Montauti M and Wezel F (2016) Charting the Territory: Recombination as a Source of Uncertainty for Potential Entrants. Org. Science. Vol. 27(4): 954-971

Navis C and Glynn MA (2010) How New Market Categories Emerge: Temporal Dynamics of Legitimacy, Identity, and Entrepreneurship in Satellite Radio, 1990-2005. Adm. Science Quar. 55(3): 439-471

Negro, G., Hannan M. T. and Rao. H. 2010. Categorical Contrast and Niche Width: Critical Success in Winemaking. I. and Corporate Change 19: 1397-1425.

Negro G and Leung MD (2013) “Actual” and Perceptual Effects of Category Spanning. Org. Science, 24 (3): $684-696$.

Oppenheimer D, Meyvis T and Davidenko N (2009) Instructional Manipulation Checks: Detecting Satisficing to Increase Statistical Power. J. of Expe. Social Psych. 45, 867-872

Paolella L and Durand R (2016) Category spanning, evaluation, and performance: Revised theory and test on the corporate law market. Acad. of Management J., 59: 330-351.

Pavelchak MA (1989) Piecemeal and category-based evaluation: An idiographic analysis.' J. of Personality and Social Psych. 56, 354-363

Pontikes EG (2012) Two sides of the same coin: How ambiguous classification affects multiple audience evaluations. Admin. Sci. Quart. 57: 81-118.

Pontikes EG and Barnett WP (2015) The Persistence of Lenient Market Categories. Org. Science. 26: 1415-1431.

Ratneshwar S, Pechmann C and Shocker AD (1996). Goal-derived categories and the antecedents of across-category consideration. Jour. of Consumer Res. 23, 240-250.

Ratneshwar S, Barsalou LW, Pechmann C and Moore M (2001). Goal-derived categories: The role of personal and situational goals in category representations. Jour. of Cons. Psych. 10(3), 147-157.

Reips UD (2002) Standards for Internet-based experimenting. Expe. Psych. 49, 243-256

Rosa JA, Porac JF, Runser-Spanjol J and Saxon MS (1999) Sociocognitive dynamics in a product market. J. of Market. 63, 64-77

Rosch E and Mervis CB (1975) Family resemblances: Studies in internal structure of categories. Cog. Psych. 7: 573-605

Roy DP and Cornwell TB (2004) The effects of consumer knowledge on responses to event sponsorships. Psych. and Marketing, 21 (3), 185-207

Smith ES and Chae H (2017). The effect of organizational atypicality on reference group selection and performance evaluation. Org. Science. 28(6): 1134-1149

Sharkey AJ (2014) Categories and Organizational Status: The Role of Industry Status in the Response to Organizational Deviance. Amer. Journal of Soc. 119(5): 1380-1433

Suarez FS, Grodal S and Gotsopoulos A (2015) Perfect Timing? Dominant Category, Dominant Design and the Window of Opportunity for Firm Entry. Strategic Management J. 36(3): 437-448.

Tripsas M (2009) Technology, identity, and inertia through the lens of the digital photography company. Org. Science. 20(2): 441-460.

Vannette DL (2016) Testing the Effects of Different Types of Attention Interventions on Data Quality in Web Surveys. Experimental Evidence From a 14 Country Study. Paper presented at the 71st Annual Conference of the American Association for Public Opinion Research in Austin, TX

Vergne JP and Wry T (2014) Categorizing categorization research: Review, integration, and future directions. J. of Management Stud. 51(1): 56-94

Zhao EY, Fisher G, Lounsbury M and Miller D (2017) Optimal distinctiveness: Broadening the interface between institutional theory and strategic management. Strategic Management J. 38(1): 93-113.

Zhao EY, Masakazu I, Devereaux Jennings P and Lounsbury M (2018) Optimal distinctiveness in the console video game industry: An exemplar-based model of proto-category evolution Org. Science. 29(4): 588-611

Wry T, Lounsbury M and Devereaux Jennings P. (2014). Hybrid vigor: securing venture capital by spanning categories in nanotechnology. Acad. of Management J. 57: 1309-1333

Wu LL and Barsalou LW (2009). Perceptual simulation in conceptual combination: Evidence from property generation. Acta Psychologica, 132: 173-189

Zuckerman EW (2017) The categorical imperative revisited: Implications of categorization as theoretical tool. In R. Durand, N. Granqvist, \& A. Tyllstrom" (Eds.), Categories, categorization and categorizing: Category studies in sociology, organizations and strategy at the crossroads (Vol. 51). Research in the Sociology of Organizations. Bingley, UK: Emerald Group Publishing Limited. 


\begin{tabular}{|c|c|c|c|c|c|c|}
\hline STUDIES & 1 & $\mathbf{A}$ & $\mathbf{B}$ & 2 & $\mathbf{C}$ & $\mathbf{D}$ \\
\hline Objective & $\begin{array}{l}\text { Testing H1-does } \\
\text { priming GBC* } \\
\text { positively impact the } \\
\text { evaluation of an } \\
\text { ambiguous offering? }\end{array}$ & $\begin{array}{l}\text { Do evaluators use } \\
\text { central tendency to } \\
\text { discount an ambiguous } \\
\text { offering when placed in } \\
\text { a control group or } \\
\text { primed with PBC? }\end{array}$ & $\begin{array}{l}\text { Do the findings of } \\
\text { Study } 1 \text { replicate } \\
\text { with a real-world } \\
\text { population of } \\
\text { entrepreneurs? }\end{array}$ & Testing $\mathrm{H} 1$ and $\mathrm{H} 2$ & $\begin{array}{l}\text { Do the results of Study } \\
2 \text { replicate if with an } \\
\text { increased unclear } \\
\text { attribute as used in } \\
\text { Study 2? }\end{array}$ & $\begin{array}{l}\text { Do the results of Study } \\
2 \text { replicate if we } \\
\text { introduce a different } \\
\text { unclear product } \\
\text { attribute? }\end{array}$ \\
\hline Who? & 320 M-Turkers & 199 Students (lab) & $\begin{array}{l}142 \text { Entrepreneurs } \\
\text { (field) }\end{array}$ & 317 M-Turkers & 160 M-Turkers & 319 M-turkers \\
\hline Product & loan & loan & loan & smartphone & smartphone & smartphone \\
\hline DV & $\begin{array}{l}\text { Likability measure } \\
\text { (10-point scale) }\end{array}$ & Same as in Study 1 & Same as in Study 1 & $\begin{array}{c}\text { Likability measure }+ \\
\text { perceived market success } \\
\text { (both } 10 \text {-point scale) }\end{array}$ & Same as in Study 2 & Same as in Study 2 \\
\hline $\begin{array}{l}\text { IVs } \\
\text { (manip.) }\end{array}$ & $\begin{array}{c}\text { Ambiguity: } \\
\text { Introduction of } \\
\text { multiple unclear } \\
\text { attributes } \\
\text { Categorization } \\
\text { processes: Control } \\
\text { vs. PBC** vs. GBC }\end{array}$ & $\begin{array}{c}\text { Ambiguity: Same as in } \\
\text { Study } 1 \\
\text { Categorization } \\
\text { processes: Control vs. } \\
\text { PBC (manipulated via } \\
\text { similarity-based } \\
\text { judgment) }\end{array}$ & $\begin{array}{l}\text { Ambiguity: Same } \\
\text { as in Study } 1 \\
\text { Categorization } \\
\text { processes: PBC vs. } \\
\text { GBC }\end{array}$ & $\begin{array}{c}\text { Ambiguity: } \\
\text { Introduction of one } \\
\text { unclear attribute } \\
\text { Categorization } \\
\text { processes: Control vs. } \\
\text { PBC vs. GBC }\end{array}$ & Same as in Study 2 & Same as in Study 2 \\
\hline $\begin{array}{l}\text { Main } \\
\text { results }\end{array}$ & $\begin{array}{l}\text { Participants evaluate } \\
\text { negatively an } \\
\text { ambiguous offering } \\
\text { when placed in a } \\
\text { control group or } \\
\text { primed with PBC } \\
\text { (baseline) } \\
\text { Participants evaluate } \\
\text { positively an } \\
\text { ambiguous offering } \\
\text { when GBC is primed }\end{array}$ & $\begin{array}{l}\text { Participants evaluate } \\
\text { negatively an ambiguous } \\
\text { offering when placed in } \\
\text { a control group or } \\
\text { primed with a PBC and } \\
\text { they activate the } \\
\text { cognitive mechanism of } \\
\text { central tendency to do so }\end{array}$ & Same as in Study 1 & $\begin{array}{l}\text { Same as in Study } 1 \\
\text { The perceived utility of } \\
\text { the unclear attribute } \\
\text { mediates the positive } \\
\text { relationship between } \\
\text { GBC and the evaluation } \\
\text { of an ambiguous } \\
\text { offering }\end{array}$ & $\begin{array}{c}\text { Same as in Study } 2 \\
+ \text { Knowledgeability } \\
\text { positively moderates the } \\
\text { mediation of the } \\
\text { perceived utility of the } \\
\text { unclear attribute on the } \\
\text { relationship between } \\
\text { GBC and the evaluation } \\
\text { of the ambiguous } \\
\text { offering }\end{array}$ & $\begin{array}{l}\text { Same as in Study } 2 \\
+ \text { Same as in Study C }\end{array}$ \\
\hline
\end{tabular}

Note: in blue, studies fully presented in paper; in white, studies in online appendix. ${ }^{*} \mathrm{GBC}$ and ${ }^{* *} \mathrm{PBC}$ stand for goal-based and prototype-based categorization 


\section{FIGURES}

Figure 1: Effect of control, prototype-based, and goal-based categorization on evaluation across ambiguity conditions (Study 1)

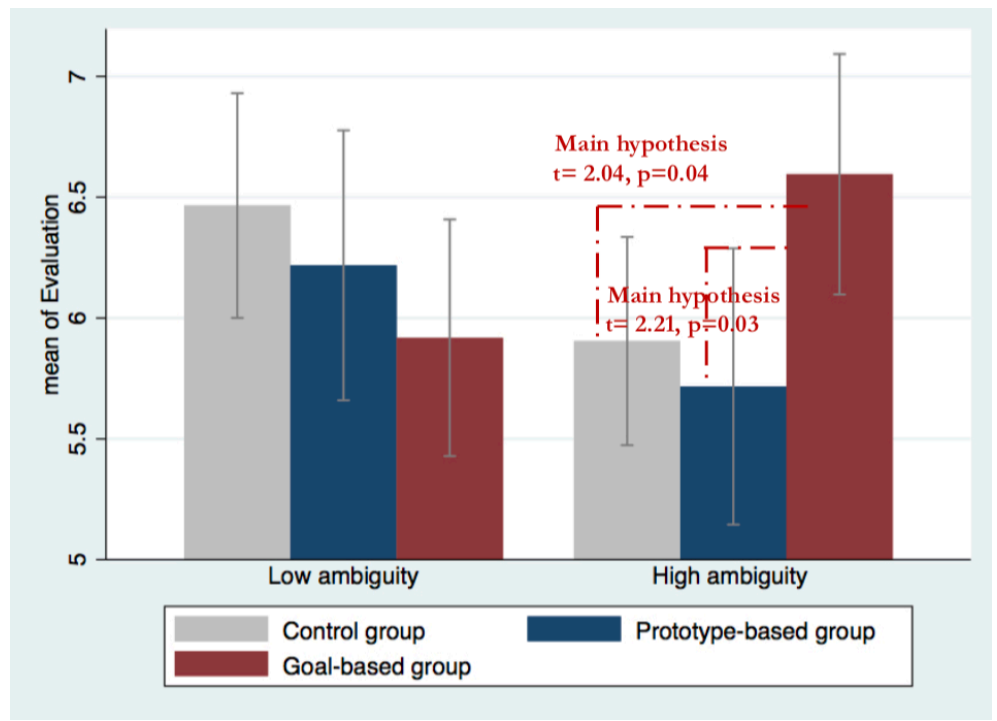

Note: The tests for $\mathrm{H} 1$ are shown graphically. $\mathrm{H} 1$ is supported because the mean of evaluation, in the highambiguity condition, is higher in the goal-based group relative to the other two groups $(M G$ oal $=6.60, \mathrm{SD}=$ 1.63 vs. $M$ Control $=5.90, \mathrm{SD}=1.73$; $t(1,103)=2.04, \mathrm{p}$ $=0.04$, two-tailed test $)$ and $(M G o a l=6.60, \mathrm{SD}=1.63 \mathrm{vs}$. $M$ prototype $=5.72, \mathrm{SD}=2.11 ; \mathrm{t}(1,93)=2.21, \mathrm{p}=0.03$, two-tailed test).

Figure 2: Effect of control, prototype-based, and goal-based categorization on evaluation across ambiguity conditions (Study 2)

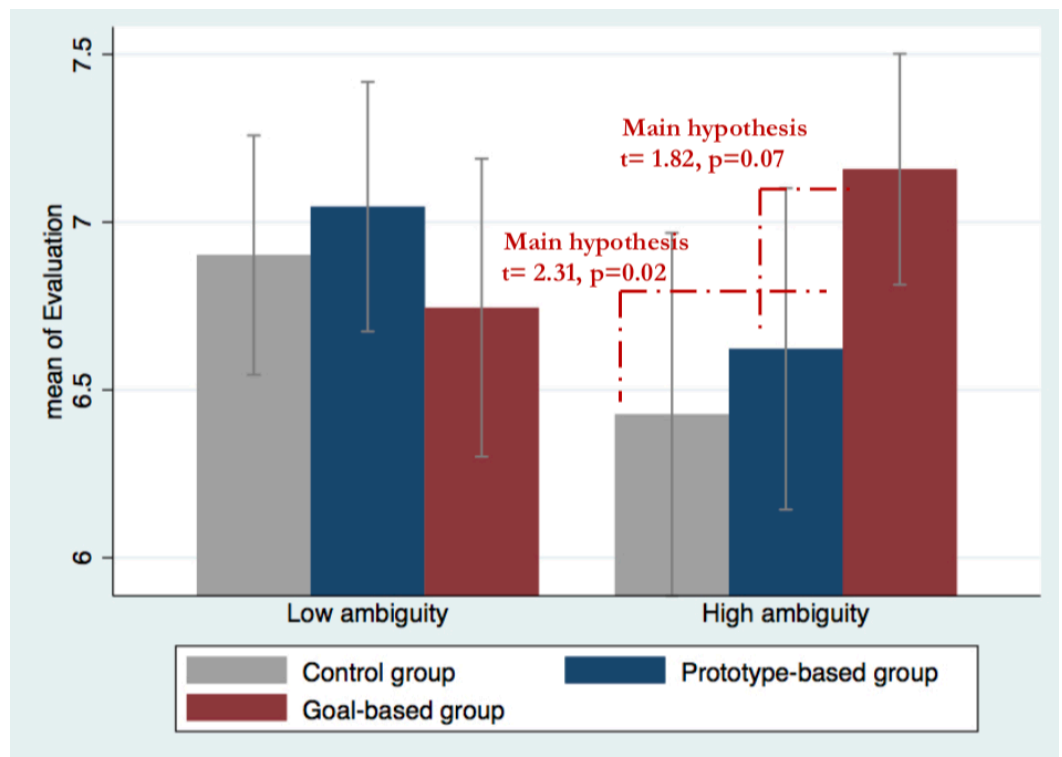

Note: The tests for H1 are shown graphically. $\mathrm{H} 1$ is supported because the mean of evaluation, in the condition of high ambiguity, is higher in the goalbased group relative to the other two groups $(M G o a l=7.15, \mathrm{SD}=1.32$ vs. $M$ Control $=6.43$, $\mathrm{SD}=1.90 ; t(1,103)=2.31, \mathrm{p}=0.02$, two-tailed test $)$ and $(M G o a l=7.15, \mathrm{SD}=1.32$ vs.

$M$ prototype $=6.62, \mathrm{SD}=1.70 ; \mathrm{t}(1,104)=1.82, \mathrm{p}$ $=0.07$, two-tailed test). 


\section{Figure 3: Mediation via Perceived Utility of the Unclear Attribute (Study 2)}

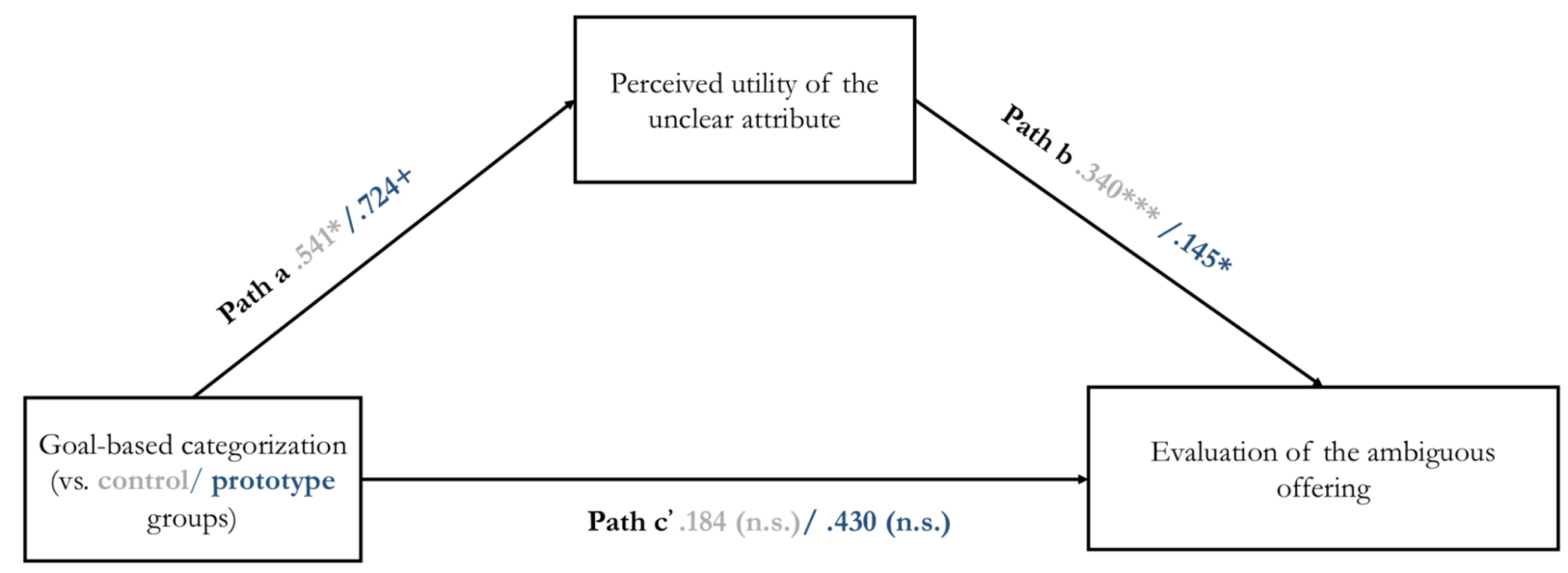

NOTE. $* \mathrm{p}<.05 ; * * \mathrm{p}<.01 ; * * * \mathrm{p}<.001$

Note: The figure shows that priming goal-based categorization (relatively to being placed in a control condition or being primed on prototype-based categorization) leads to increase the perceived utility of the unclear attribute (path a: beta $1=$ $0.541, \mathrm{p}=0.01$, CI $[0.11 ; 0.97]$ beta $_{2}=0.724, \mathrm{p}=0.09$, CI $\left.[-0.11 ; 1.56]\right)$, which in return positively impacts the evaluation of the ambiguous offering (path $b ;$ beta $_{1}=0.340, p=0.000$, CI $[0.214 ; 0.467]$; beta $2=0.145, p=0.03$, CI $[0.01 ; 0.28])$. The mediation is strong enough to cancel out the direct effect of goal-based categorization on the evaluation of the ambiguous offering. 


\section{APPENDICES}

\section{Appendix 1- Offering attributes: Loan (Study 1)}

\section{Low ambiguity condition}

Grow your business through the acquisition of new offices and new software

Loan without collateral on either the firm's or the entrepreneur's assets

Fixed rate loan

A duration of 7 years

Amount borrowed: $€ 300,000$ to $€ 5$ million

\section{High ambiguity condition}

Grow your business through tangible and intangible investments

Loan without collateral on the assets- to be determined according to investment type

Fixed or floating rate loan

A duration of 4-7 years

Amount borrowed: $€ 300,000$ to $€ 5$ million

\section{Appendix 2- Offering attributes: Consumer Good (Study 2)}

\section{Low ambiguity condition}

$4 \mathrm{G}$ surfing speed $(6 \mathrm{GHz})$, perfect for optimal web browsing

One of the best displays available on the market

Capture memories with one of the finest camera phones available today

One of the fastest smartphone processors on the market

Plenty of space (100 GB) to store your video clips or photos

\section{High ambiguity condition}

$4 \mathrm{G}$ surfing speed with floating capacity of plus or minus 3 to $9 \mathrm{GHz}$

One of the best displays available on the market

Capture memories with one of the finest camera phones available today

One of the fastest smartphone processors on the market

Plenty of space (100 GB) to store your video clips or photos 


\section{ON-LINE APPENDIX}

We present a set of four distinct experiments that provide additional for the results obtained in the context of Studies 1-2 (see Table 1 in paper for a short description).

The first two experiments (Studies A and B) are using the same financial product tested in Study 1. Study A was run in the lab with students and shows that without priming an ad hoc goal, students discount the ambiguous offering (relatively to a non-ambiguous offering). Study B replicates the findings of Study 1 with a population of real-world entrepreneurs, thus increasing the ecological validity of our findings.

The last two experiments (Studies C and D) were run on M-Turk and test the consumer good used for Study 2. Compared to Study 2, Study C increases the level of ambiguity of the offering (same unclear product attribute used in the context of Study 2: "4G surfing speed"). Study D replicates the findings of Study 2 by manipulating a different product attribute (the "display" vs. "4G surfing speed"). In Studies C and $\mathrm{D}$, we test whether knowledgeable evaluators are more likely to see a higher utility in unclear attributes than novice evaluators when goal-based categorization is primed.

Study A: Do evaluators use central tendency to discount ambiguous offerings when not primed on goal-based categorization?

In this study, we show that when participants are primed with prototype-based categorization or placed in a control condition, they activate the cognitive mechanism of central tendency and they severely discount ambiguous offerings. These results explain why, in Studies 1 and 2, for the participants placed in a control group or primed with a prototype, they discount ambiguous product offerings.

Participants. We partnered with an experienced behavioral lab located in a metropolitan European city to recruit participants. The behavioral lab was set up in 2002 and has been used as a resource in more than 110 peer-reviewed articles (including, among others, articles published in Psychological Science, Journal of Consumer Psychology, and PNAS). In this study, students were instructed to imagine themselves as entrepreneurs evaluating an offering that could possibly help them to grow their venture. Please note that this experiment was conducted in French.

We employed a 2 (low/high ambiguity) $\times 2$ (prototype-based categorization or not) study design. One hundred ninety-nine students successfully completed the study and were randomly assigned to one of the four conditions. Upon completion of the study, we debriefed the students, and each received monetary compensation in accordance with the behavioral lab's policies. Of all participants, $40 \%$ had a high school diploma or two years of college education at most; $60 \%$ had at least a bachelor's degree or a master's degree; $69 \%$ of the participants were female; and the average age of the study population was 22.65 years $[\mathrm{SD}=3.04]$.

Procedures. Students participated in two sessions held 7 to 10 days apart. Because a student audience might discount financial products due to either their ignorance or business inexperience (Fiske and Neuberg 1990, p. 5). The first session served as a pretest to introduce participants to the financial products being tested and five attributes of each. In this pretest, we asked participants to randomly evaluate each attribute in isolation. These evaluations helped us to build an ad hoc measure of central tendency that computed each participant's average liking of the five attributes. This measure of central tendency, which is similar to the "difference from average" variable used by Zhao and colleagues (2018, p. 605), enabled us to capture whether any difference existed between the average liking of the five attributes and the evaluation of the ambiguous loan during session 2. Finally, in this pretest, we also collected several control variables: gender, educational background, levels of education, and age. Seven to 10 days after the completion of 
session 1, participants attended a second session, where they were randomly assigned to one of the four conditions (low/high ambiguity $\times$ prototype-based categorization/no priming).

Level of Ambiguity. We used the same manipulation of ambiguity as per Study 1.

Prototype-Based Categorization vs. Control. In this first study, we compared a control group with a prototype-based group that we primed using a manipulation that favors similarity-based judgments (Pavelchak 1989, Kopetz and Kruglanski 2008 - Study 1). Just before they evaluated an offering, the primed participants were asked to classify the offering as "a loan," a "capital investment," a "mix of different financial product categories," or "I don't know." In the control condition, however, participants immediately evaluated the offering without first having been asked the classification question, so as to not induce any prototypical-based judgments when evaluating the offering (Pavelchak 1989, p. 357).

Dependent variable. Consistent with Study 1, participants were asked to evaluate the loan on a 10-point scale from I do not like it at all (0) to I like it very much (9). Across conditions, evaluation had a mean $=5.77$ $(\mathrm{SD}=1.90)$. This evaluation score was the dependent variable in our analyses of variations (ANOVAs). Manipulation Check. Using our perceived ambiguity score (consistent with Study 1), we checked and confirmed that participants found the offering significantly more ambiguous $(\mathrm{M}=5.31, \mathrm{SD}=2.14)$ in the condition of high ambiguity than in the condition of low ambiguity $(\mathrm{M}=3.90, \mathrm{SD}=2.15) \mathrm{t}(1,197)=4.59$, $\mathrm{p}=0.000$, two-tailed test.

Testing prototype vs. control on evaluation. First, we conducted a one-way ANOVA (low vs. high ambiguity) on the evaluation. As expected, the evaluation was higher in the low-ambiguity condition than in the high-ambiguity condition $\left(M_{\text {Low ambiguity }}=6.25, \mathrm{SD}=1.66\right.$ vs. $M_{\text {high ambiguity }}=5.25, \mathrm{SD}=2.02$; $t(1$, $197)=3.79, \mathrm{p}=0.000$, two-tailed test). This result shows that participants severely discounted the ambiguous offering compared with the non- ambiguous offering, irrespective of whether participants were placed in the prototype-based group or a control group.

Second, we performed a 2 (high ambiguity vs. low ambiguity) $\times 2$ (prototype-based vs. control) ANOVA on the likability measure to test whether we observed any differences when participants were placed in the prototype or control groups. As expected, the interaction effect was not significant $(\mathrm{F}(1,195)$ $=0.36, \mathrm{p}=0.55)$, and the evaluation of the ambiguous offering did not differ across the prototype and control groups $\left(M_{\text {prototype }}=5.29, \mathrm{SD}=1.81\right.$ vs. $M_{\text {control }}=5.22, \mathrm{SD}=2.21 ; t(1,92)=0.15, \mathrm{p}=0.88$, twotailed test).

Finally, we provide evidence that participants evaluated the ambiguous loan using central tendency. During session 1, participants separately evaluated each unclear attribute of the ambiguous loan on a 10point scale, and we created a score based on the average liking of these five attributes. Across conditions, the average likability of the five unclear loan's attributes was $M_{\text {score average liking }}=5.13, \mathrm{SD}=1.17$. This score did not significantly differ from the mean of the evaluated ambiguous loan, either in the prototype-based $\operatorname{group}\left(M_{\text {score average liking }}=5.24, \mathrm{SD}=1.27\right.$ vs. $M_{\text {ambiguous loan }}=5.30, \mathrm{SD}=1.80 ; \mathrm{p}=0.81$, two-tailed test $)$ or in the control group $\left(M_{\text {score average liking }}=5.02, \mathrm{SD}=1.07\right.$ vs. $M_{\text {ambiguous loan }}=5.22, \mathrm{SD}=2.21 ; \mathrm{p}=0.56$, twotailed test). If the participants did not rely on central tendency in both the prototype-based and control groups, we should have observed a difference across subgroups between the average liking of the five attributes made during session 1 and the evaluation of the ambiguous loan during session 2 . Our finding of no significant difference provides evidence that, irrespective of the groups in which participants were placed (prototype-based vs. control), they used central tendency to evaluate the ambiguous product offering. Said differently, central tendency is the baseline mechanism of entity evaluation.

This study thus supports the idea that when participants are placed in either a control condition or a prototype-base group, they rely on central tendency and severely discount an ambiguous product. 
In this study, we show that the positive effect of goal-based categorization on the evaluation of the ambiguous business loan used for Study 1 and how it replicates with a population of real-word entrepreneurs, who were Public Bank's initial target group of this product offering.

Participants. Most participants, recruited as a result of our partnership with Public Bank, were entrepreneurs who were customers of the bank and operated small- to medium-sized companies. We complemented these participants with alumni of a master's in entrepreneurship program from a top-tier European business school, all of whom had created a business venture. All participants in this Study were entrepreneurs from the same country, and all spoke the same language (French). One hundred forty-two entrepreneurs completed the study. Consistent with Study 1, because this Study was conducted online, we used an IMC to capture the participants' level of attention (Oppenheimer et al. 2009, p. 867). Forty participants did not pass the attention check and were not excluded; however, excluding these participants did not change the results presented in the analyses below. Of all participants, 93\% participants were male, and the average age of the total study population was 34.28 years [SD $=7.63$ ].

Procedures. Because we knew before running the experiment that the pool of potential participants was limited, we restricted our analysis to prototype-based versus goal-based, and we used the same manipulations for ambiguity and for prototype-based vs. goal-based as per Study 1.

Measures. Consistent with Study 1, we measured evaluation as a likability score on a scale ranging from $I$ do not like it at all (0) to I like it very much (9). However, to take advantage of our audience being entrepreneurs and to go beyond the evaluation of likability, which does not engage many respondents, we added a question more specific to their interests, centered on the relevance of the offering for entrepreneurs: "Do you think it would be relevant to offer this product to other entrepreneurs in the future?" with possible answers ranging from not at all (0) to absolutely (9). As the correlation across answers between the likability and the relevance items was high $(\mathrm{r}=0.76, \mathrm{p}<0.01)$, we averaged these two variables to create a second evaluation score: Relevance.

Manipulation Check. Consistent with Study 1, participants found the offerings in the high-ambiguity condition to be significantly more ambiguous $(\mathrm{M}=4.14, \mathrm{SD}=2.65)$ than in the low-ambiguity condition $(\mathrm{M}=3.34, \mathrm{SD}=2.72) \mathrm{t}(1,140)=1.77, \mathrm{p}=0.07$, two-tailed test.

Testing the Hypotheses. We performed a 2 (low ambiguity vs. high ambiguity) $\times 2$ (prototype-based vs. goal-based categorization) ANOVA on the likability measure to test whether any observable difference emerged across our subgroups. The two-way interaction was significant $(F(1,138)=5.40, p=0.02)$, which directly supports our main hypothesis.

First, the results show that participants severely discounted the ambiguous offering when placed the prototype-based group $\left(M_{\text {High ambiguity }}=6.2, \mathrm{SD}=1.82\right.$ vs. $M_{\text {Low ambiguity }}=7.49, \mathrm{SD}=1.73 ; t(1,67)=$ $2.99, \mathrm{p}=-0.004$, two-tailed test ). This result is consistent with the results from Study 1 and Study A. Second, in the high-ambiguity condition, the mean of our likability measure was significantly higher in the goal-based group than in the prototype-based group $\left(M_{\text {Goal }}=7.09, \mathrm{SD}=1.71 \mathrm{vs} . M_{\text {prototype }}=6.2, \mathrm{SD}=\right.$ $1.82 ; t(1,60)=1.99, \mathrm{p}=0.05$, two-tailed test), providing support for H1. Furthermore, in the lowambiguity condition, the mean of our likability measure was lower in the goal-based group than in the prototype-based group $\left(M_{\text {Goal }}=6.97, \mathrm{SD}=1.86\right.$ vs. $M_{\text {Prototype }}=7.49, \mathrm{SD}=1.73 ; t(1,78)=1.27, \mathrm{p}=-0.2$, two-tailed test); however, this result does not reach significance.

\section{Confirmatory Tests: Relevance and Complexity}

In our confirmatory tests, we first ran the same 2 (low ambiguity vs. high ambiguity) $\times 2$ (prototype-based vs. goal-based categorization) ANOVA using the relevance measure instead of the likability measure. The two-way interaction remained significant $(F(1,138)=3.91, \mathrm{p}=0.05)$, which corroborates the results found 
with likability. Second, consistent with Study 1, we ensured that our manipulation of prototype categorization vs. goal-based categorization did not influence participants to value greater complexity. To do so, we ran a 2 (high ambiguity vs. low ambiguity) $\times 2$ (prototype-based vs. goal-based categorization) ANOVA on the perception of complexity, and the interaction was not significant $(\mathrm{F}(1,138)=0.01, \mathrm{p}=$ 0.92). In addition, we compared the mean of this complexity variable across conditions of ambiguity, and we were unable to detect an effect at $\mathrm{p}<0.1$. Consistent with Study 1, to ensure that our priming on a goal did not induce a different thinking process among participants, we measured, in seconds, the time that participants spent on reading (i) the manipulation question and (ii) the offering. In both cases the interaction was not significant $(\mathrm{F}(1,138)=1.12, \mathrm{p}=0.29)$ and $(\mathrm{F}(1,138)=0.55, \mathrm{p}=0.46)$, respectively.

\section{Effect of prototype-based and goal-based categorization on evaluation across ambiguity conditions (Replication study with entrepreneurs)}

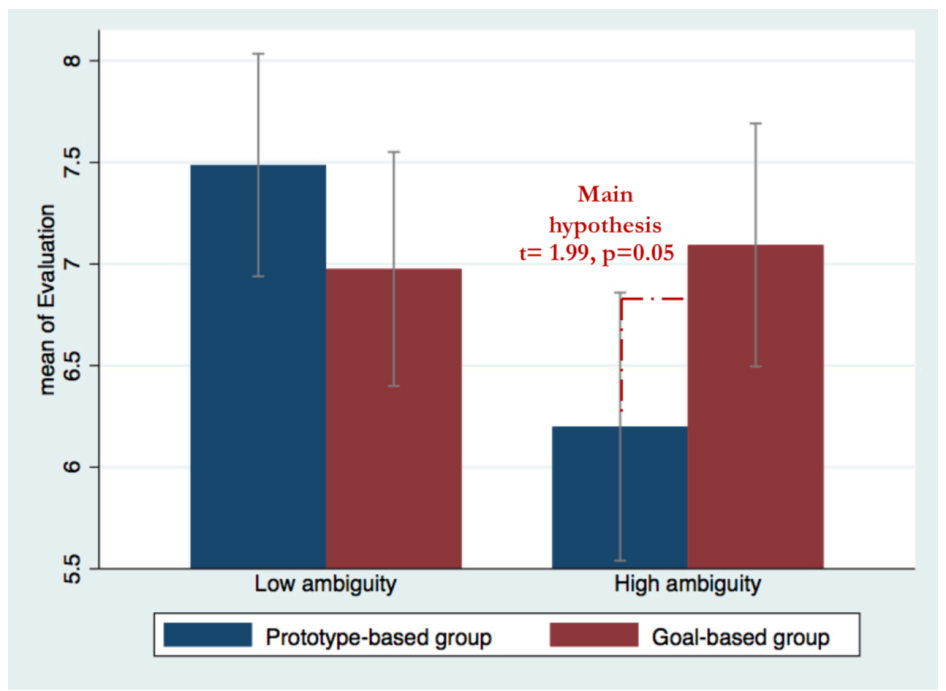

Note: The test for $\mathrm{H} 1$ is shown graphically. $\mathrm{H} 1$ is supported because the mean of evaluation, in the high-ambiguity condition, is higher in the goal-based group than in the prototype-based group $\left(\mathrm{M}_{\text {Goal }}=7.09, \mathrm{SD}=\right.$ 1.71 vs. $\mathrm{M}_{\text {Prototype }}=6.2, \mathrm{SD}=1.82 ; \mathrm{t}(1,60)$ $=1.99, \mathrm{p}=0.05$, two-tailed test).

\section{Study C: Increasing the Degree of Ambiguity (Study 2)}

In this Study, we turn to M-Turk to increase the ambiguity of the product by decreasing the level of clarity of the attribute manipulated in Study 2 (" $4 \mathrm{G}$ surfing speed"). Consistent with the findings reported in Study 2, we pretested that participants struggled to associate this attribute with the smartphone product category. Finally, we tested and found that knowledgeable evaluators were more capable and likely to see a higher perceived utility in unclear attributes (compared to novice participants).

Participants: One hundred and sixty participants were placed either in a control, prototype-based or goalbased group and tasked to evaluate the ambiguous offering. Of all participants, $46 \%$ of the participants were female and the average age of the study population was 39.18 years [SD $=13.27$ ].

Procedure: Manipulation of prototype-based vs. goal-based categorization is the same than the one used in Study 2.

Level of ambiguity: To decrease the level of clarity of the unclear attribute, we introduced the following unclear attribute " $4 \mathrm{G}$ surfing speed with floating rate capacity of $4 \mathrm{x}$ to $4+3 \mathrm{GHz}$ " instead of " $4 \mathrm{G}$ surfing speed with floating capacity of plus or minus 3 to $9 \mathrm{GHz}$ ", all other product attributes remain identical than the ones presented in Study 2. 
Measures: We used the same measures than the ones reported in Study 2. Note that we use the evaluation score framed in a third-order way in the analysis below, even if results are consistent when the scores are not framed in a third-order way, although with less statistical power.

Manipulation Checks. Before running our experiment, we ran a pretest on M-Turk with 78 participants-44\% participants were male, and the average age was 39.71 years [SD $=11.82]$ - to investigate whether participants placed in the high-ambiguity condition perceived the offering as being more ambiguous and whether they ability to associate the unclear attribute with the smartphone product category was impaired by our ambiguity manipulation. Using the same score presented in previous studies to capture perceived ambiguity, we found that participants perceived the offerings to be significantly more ambiguous $(\mathrm{M}=4.5, \mathrm{SD}=2.28)$ in the high-ambiguity condition than in the low-ambiguity condition ( $\mathrm{M}$ $=3.5, \mathrm{SD}=2.47) \mathrm{t}(1,76)=1.91, \mathrm{p}=0.06$, two-tailed test). In addition, participants were more likely to associate the clear product attribute ("4G surfing speed $(6 \mathrm{GHz})$, perfect for optimal web browsing") with the smartphone product category than the unclear attribute, $(\mathrm{M}=7.73, \mathrm{SD}=1.35)$ for clear product attribute compared to $\mathrm{M}=5.78, \mathrm{SD}=2.67), \mathrm{t}(1,76)=4.07, \mathrm{p}=0.000$, two-tailed test, for the unclear attribute.

\section{Testing H1: prototype-based vs. goal-based groups}

When evaluating the ambiguous offering, the mean of evaluation was significantly higher in the goal-based group than in the prototype-based group $\left(M_{\text {Goal }}=7.49, \mathrm{SD}=1.11\right.$ vs. $M_{\text {prototype }}=6.73, \mathrm{SD}=1.93 ; t(1,99)$ $=2.46, \mathrm{p}=0.02$, two-tailed test), thereby supporting our main hypothesis.

Testing H2: mediation via perceived utility of the unclear attribute.

Using the PROCESS model as per Study 2, we found that goal-based categorization had a positive effect on the perceived utility of the unclear attribute (path a; beta $=0.708, \mathrm{p}=0.04, \mathrm{CI}[0.12 ; 1.40]$ ), and, perceived utility has, in turn, a positive effect on the evaluation of the ambiguous offering (path b; beta $=$ $0.405, \mathrm{p}=0.000$, CI [0.25; 0.56]). Finally, a bias-corrected bootstrap based on 10,000 bootstrap samples showed that the interval for the indirect effect was entirely above zero when comparing goal-based categorization to a prototype-based groups (0.007-0.728).

\section{Confirmatory Test: The Effect of Knowledgeability}

We then investigated whether knowledgeability impacted our hypothesized mechanism. To measure participant knowledgeability, after collecting our main scores, we asked four multiple-choice questions that directly tested participants' knowledge of the smartphone category. Each question had four answer choices, only one of which was correct. To measure participant knowledgeability, we created a binary variable based on the median number of correct responses to these questions. In this analysis, the median value is 3; thus, participants were considered to be knowledgeable when they answered at least three questions correctly, making the knowledgeability variable worth 1 , and 0 otherwise.

Testing the effect of knowledgeability on the mediation of perceived utility of the unclear attribute is equivalent to computing an index of moderated mediation (Hayes 2015, p. 2). We used the PROCESS macro in SPSS software, developed by Hayes, to run this statistical model, contrasting the goal-based condition with the prototype-based condition. The interaction between goal-based categorization and knowledgeability on the perceived utility of the unclear attribute was positive and marginally significant (beta coefficient $=1.527, \mathrm{p}=0.07$, CI $[-0.13 ; 3.18]$ ) and the conditional effect of goal-based categorization on the perceived utility of the unclear product attribute when participants are knowledgeable (i.e., knowledgeability $=1$ ) is positive and significant (beta coefficient $=2.036, \mathrm{p}=0.008$, CI [0.56; 3.51]). This result is consistent with our expectations that knowledgeable evaluators see more utility in an unclear product attribute when goal-based categorization is primed (relatively to prototype-based categorization). Finally, a bias-corrected bootstrap based on 10,000 bootstrap samples showed that the interval for the indirect effect was entirely above zero (0.145-1.657) for the knowledgeable subgroup. 


\section{Effect of prototype-based and goal-based categorization on evaluation across ambiguity conditions (Replication study of Study 2)}

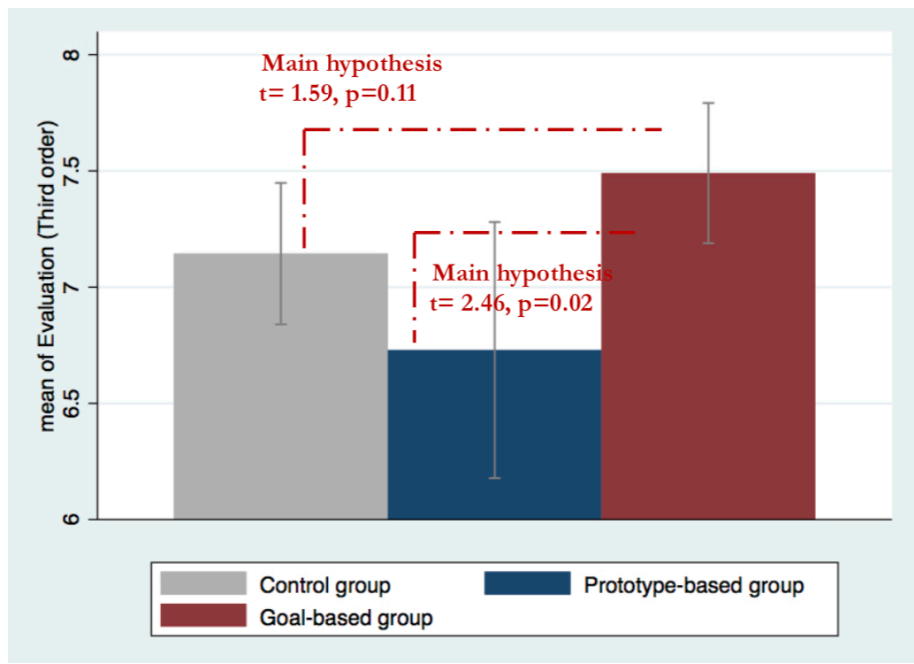

Note: The test for $\mathrm{H} 1$ is shown graphically. $\mathrm{H} 1$ is supported because the mean of evaluation is higher in the goal-based group than in the prototype-based group $\left(\mathrm{M}_{\mathrm{Goal}}=7.49, \mathrm{SD}=\right.$ 1.11 vs. $\mathrm{M}_{\text {Prototype }}=6.73, \mathrm{SD}=1.93 ; \mathrm{t}(1,99)=$ 2.46, $\mathrm{p}=0.02$, two-tailed test).

\section{Mediation via Perceived Utility of the Unclear Attribute (Replication of Study 2)}

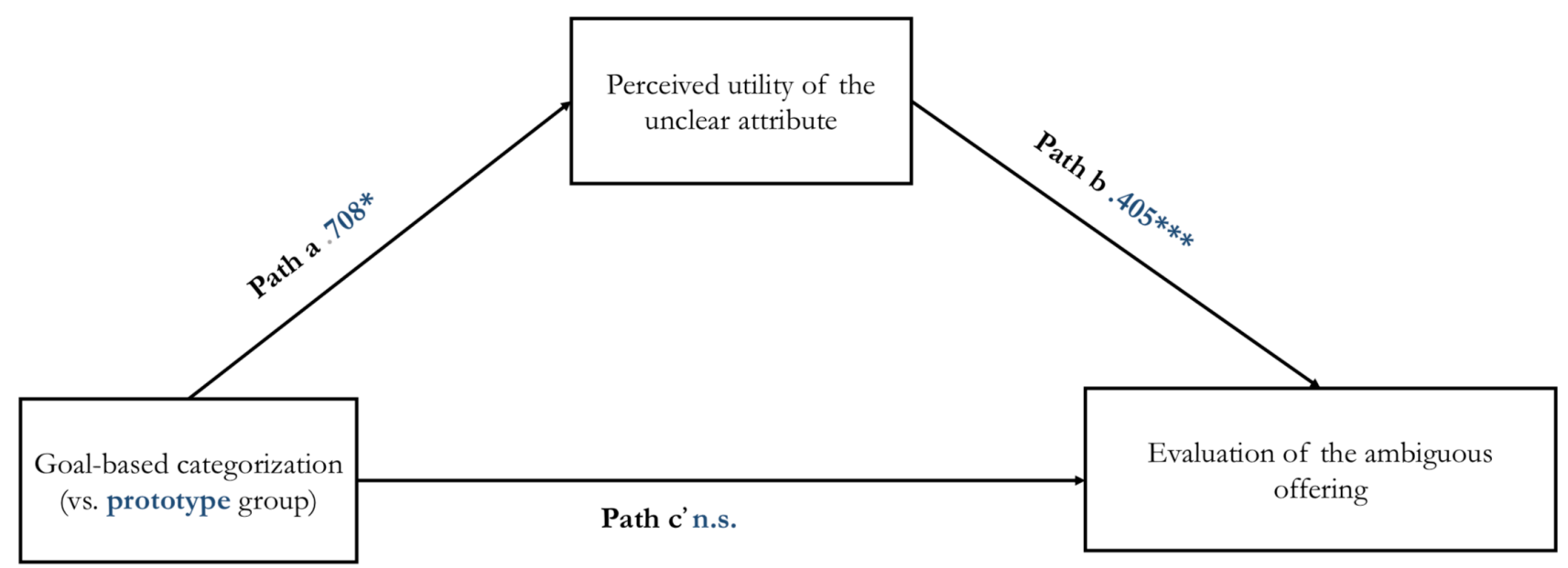

NOTE. $* \mathrm{p}<.05 ; * * \mathrm{p}<.01 ; * * * \mathrm{p}<.001$

Note: The figure shows that priming goal-based categorization (relatively to being primed on prototype-based categorization) leads to increase the perceived utility of the unclear attribute (path a; beta $=0.708, \mathrm{p}=0.04$, CI $[0.12$; 1.40]) which in return positively impacts the evaluation of the ambiguous offering (path $\mathrm{b}$; beta $=0.405, \mathrm{p}=0.000, \mathrm{CI}$ $[0.25 ; 0.56])$. The mediation is strong enough to cancel out the direct effect of goal-based categorization on the evaluation of the ambiguous offering. 


\section{Study D: Using a Different Unclear Product Attribute (Study 2)}

Consistent with Study 2, we turn to M-Turk to run another study manipulating a different product attribute. Therefore, we manipulated the display attribute and we pretested that participants struggled to associate the unclear attribute with the smartphone product category. Finally, as in Study C, we tested and found that knowledgeable evaluators were more likely to see a higher perceived utility in unclear attributes (compared to novice participants).

Participants. Consistent with Study 2, participants were recruited on M-Turk, were asked to imagine that they were buying a new phone. Similar to Study 2, participants were randomly assigned to a 2 (high/low ambiguity) $\times 3$ (control vs. prototype-based vs. goal-based) study design. Three-hundred nineteen participants, receiving financial compensation, completed the study. Twenty-six participants failed this attention test; and, consistent with recent guidelines, we did not exclude them. However, their exclusion did not change the significance of the results reported below. Of all participants, 55\% were male, and the average age was 32.21 years $[\mathrm{SD}=10.22]$.

\section{Level of ambiguity.}

Consistent with Studies 1-2, each product offering across the low- and high-ambiguity conditions presented five product attributes. Consistent with study 2, we introduced only one unclear attribute, holding constant other product attributes presented across conditions. Contrary to Study 2, we manipulated the attribute related to the display. As such, participants in the low-ambiguity condition read that the product offering has "one of the best displays available for a mobile phone," whereas participants in the high-ambiguity condition read that the product offering has "one of the best displays with plus or minus 1400:1 contrast ratio."

Priming Prototype vs Goal-Based Categorization. We used the same priming procedure than the one presented in Study 2.

Measures. We used the same combined measure of evaluation presented in Study 2 (Gibbert and Mazursky 2009, p. 655). In this study, our evaluation score had a mean $=7.08(\mathrm{SD}=1.29)$ and was used as dependent variable. Following evaluation, consistent with Study 2, we asked participants to evaluate the utility of each feature presented in the offering.

Manipulation Checks. Before running our experiment, we ran a pretest on M-Turk with 103 participants - 58\% participants were male, and the average age was 33.17 years [SD $=10.28$ — to investigate whether participants placed in the high-ambiguity condition perceived the offering as being more ambiguous. Using the same score presented in previous studies to capture perceived ambiguity, we found that participants perceived the offerings to be significantly more ambiguous $(\mathrm{M}=6.10, \mathrm{SD}=1.91)$ in the high-ambiguity condition than in the low-ambiguity condition $(\mathrm{M}=4.98, \mathrm{SD}=2.40) \mathrm{t}(1,101)=$ $2.61, \mathrm{p}=0.01$, two-tailed test). In addition, we run another test with a different set of 82 participants-of which $62 \%$ participants were male, and the average age was 29.72 years [SD $=7.10]$. Participants were split in two groups and asked whether they would, on a 10-point scale, "immediately associate" the clear attribute ("one of the best displays available for a mobile phone") "with the smartphone product category?" or the unclear attribute ("One of the best displays with plus or minus 1400:1 contrast ratio") "with the smartphone product category?". As expected, participants were more likely to associate the clear product attribute with the smartphone product category than the unclear attribute $(\mathrm{M}=7.41, \mathrm{SD}=1.22)$ for clear product attribute compared to $(\mathrm{M}=6.68, \mathrm{SD}=1.19) \mathrm{t}(1,80)=2.74, \mathrm{p}=0.008$, two-tailed test $)$, for the unclear attribute.

\section{Testing the Hypotheses.}

First, to test whether participants' evaluation differed across conditions, we performed a 2 (high ambiguity vs. low ambiguity) $\times 3$ (control vs. prototype-based vs. goal-based categorization) ANOVA on 
evaluation $(\mathrm{F}(2,313)=4.75, \mathrm{p}=0.009)$, which shows that our evaluation score substantially differed across conditions of ambiguity and groups.

Second, we checked whether we could detect any differences across the control and prototypebased categorization groups. We ran a 2 (high ambiguity vs. low ambiguity) $\times 2$ (control vs. prototypebased categorization) ANOVA on our evaluation score. As expected, the effects did not differ across conditions and groups, and the interaction term had no significant effect $(\mathrm{F}(1,206)=0.14, \mathrm{p}=0.71)$. This result is consistent with the results of previous studies. Furthermore, without discriminating between the control and the prototype-based groups, ambiguity had a negative effect on evaluation $\left(\mathrm{M}_{\text {Low ambiguity }}=7.29\right.$, $\mathrm{SD}=.99$ vs. $\mathrm{M}_{\text {High ambiguity }}=6.84, \mathrm{SD}=1.35 ; \mathrm{t}(1,208)=2.82, \mathrm{p}=0.005$, two-tailed test $)$, which shows that both participants who were primed with a prototype and those placed in a control group significantly discounted the ambiguous mobile phone.

Third, we investigated the presence of a difference in evaluation between the goal-based categorization group and the other two groups across conditions of ambiguity. We thus ran two different ANOVAs and found that the interaction effect was significant in both cases $(\mathrm{F}(1,218)=8.01, \mathrm{p}=0.005)$ for the control group and $(\mathrm{F}(1,202)=5.12, \mathrm{p}=0.02)$ for the prototype group, which shows that evaluation differs across groups and conditions of ambiguity.

As per previous studies, it follows from the two preceding points that we should observe significant differences across groups (goal-based vs. the other two) for the high and low ambiguity conditions. As expected, in the high-ambiguity condition, the mean of evaluation was significantly higher in the goal-based group than in the control group $\left(\mathrm{M}_{\mathrm{Goal}}=7.33, \mathrm{SD}=1.15 \mathrm{vs} . \mathrm{M}_{\text {Control }}=6.87, \mathrm{SD}=1.27\right.$; $\mathrm{t}(1$, $106)=1.96, \mathrm{p}=0.05$, two-tailed test $)$ and the prototype-based group $\left(\mathrm{M}_{\text {Goal }}=7.33, \mathrm{SD}=1.15\right.$ vs. $\mathrm{M}_{\text {prototype }}$ $=6.79, \mathrm{SD}=1.44 ; \mathrm{t}(1,97)=2.06, \mathrm{p}=0.04$, two-tailed test), providing direct support for H1. Furthermore, in the low-ambiguity condition, the mean of evaluation was significantly lower in the goal-based group than in the control group $\left(\mathrm{M}_{\text {Goal }}=6.85, \mathrm{SD}=1.68\right.$ vs. $\mathrm{M}_{\text {Control }}=7.38, \mathrm{SD}=0.95 ; \mathrm{t}(1,112)=-2.05, \mathrm{p}=0.04$, two-tailed test) and the prototype-based group, although this difference as before was not significant $\left(\mathrm{M}_{\mathrm{Goal}}\right.$ $=6.85, \mathrm{SD}=1.68$ vs. $\mathrm{M}_{\text {Prototype }}=7.18, \mathrm{SD}=1.03 ; \mathrm{t}(1,105)=1.17, \mathrm{p}=-0.24$, two-tailed test). Thus, when evaluating a non-ambiguous product offering, priming a goal tends to be detrimental to evaluation, as a more automatic categorization process based on central tendency provides higher evaluation.

Consistent with previous studies, we ran a series of ANOVAs on (i) the perception of complexity, (ii) the time spent on the manipulation question, and (iii) the time spent reading the product offering. The interactions remained insignificant at $\mathrm{p}<0.1$, as per previous studies.

\section{Goal-Based Categorization, and an Unclear Attribute's Perceived Utility and Evaluation.}

Consistent with Study 2, we found that the perceived utility of the unclear attribute differed across groups $(\mathrm{F}(2,154)=3.73, \mathrm{p}=0.02)$, such that in the high-ambiguity condition, the mean of the perceived utility of the unclear attribute was significantly higher in the goal-based group than in the control group $\left(M_{\text {Goal }}=\right.$ $7.26, \mathrm{SD}=1.39$ vs. $M_{\text {Control }}=6.42, \mathrm{SD}=2.09 ; t(1,106)=2.47, \mathrm{p}=0.01$, two-tailed test $)$ or the prototypebased group $\left(M_{\text {Goal }}=7.26, \mathrm{SD}=1.39\right.$ vs. $M_{\text {prototype }}=6.44, \mathrm{SD}=1.88 ; t(1,97)=2.52, \mathrm{p}=0.01$, two-tailed test) whereas the perceived utility of the clear attribute did not differ across groups. In addition, we ran the same one-way ANOVA on the perceived utility of the remaining four clear attributes when participants were placed in the high-ambiguity condition, and the main effect turned insignificant at $\mathrm{p}<0.05$.

We next tested whether the positive effect of goal-based categorization on the evaluation of an ambiguous product offering was due to an increase in the perceived utility of the unclear product's attribute. Consequently, to test $\mathrm{H} 2$ and consistent with Study 2, we ran a mediation analysis to determine whether the perceived utility of the unclear attribute mediated the effect of goal-based categorization on evaluation. Consistent with Study 2, we ran an analysis that contrasted the goal-based group in two ways: first with "control group" and then with "prototype-based group". Goal-based categorization, compared to either group, has a positive effect on the perceived utility of the unclear attribute (path a: beta $_{1}=0.432, \mathrm{p}=$ 
0.01, CI [0.08; 0.76]; beta $_{2}=0.829, \mathrm{p}=0.01$, CI $\left.[0.17 ; 1.48]\right)$ and, perceived utility has, in turn, a positive effect on the evaluation of the ambiguous offering (path $b$; beta $1=0.322, p=0.000$, CI [0.21; 0.44]; beta $_{2}$ $=0.510, \mathrm{p}=0.000, \mathrm{CI}[0.39 ; 0.63])$. Finally, a bias-corrected bootstrap based on 10,000 bootstrap samples showed that the interval for the indirect effect was entirely above zero for both analyses $(0.031-0.258$ and 0.083-0.848, respectively). Notably, the mediation in both contrast analyses was strong enough to cancel out the direct effect of goal-based categorization on evaluation that we previously reported.

\section{Confirmatory Test: The Effect of Knowledgeability}

We run the same analysis than the one used for Study C. In this analysis, the median value of participant knowledgeability is 2; thus, participants were considered to be knowledgeable when they answered at least three questions correctly, making the knowledgeability variable worth 1, and 0 otherwise.

The interaction between goal-based categorization and knowledgeability on the perceived utility of the unclear attribute is positive and significant (beta coefficient $=0.681, \mathrm{p}=0.04, \mathrm{CI}[0.02 ; 1.34]$ ). Furthermore, the conditional effect of goal-based categorization on the perceived utility of the unclear product attribute when participants are knowledgeable (i.e., knowledgeability $=1$ ) is positive and significant (beta coefficient $=0.795, \mathrm{p}=0.001, \mathrm{CI}[0.32 ; 1.27])$. This result is consistent with our expectations that knowledgeable evaluators see more utility in an unclear product attribute when goal-based categorization is activated. Finally, a bias-corrected bootstrap based on 10,000 bootstrap samples showed that (i) the interval for the indirect effect was entirely above zero (0.088-0.455) and (ii) overall, the confidence interval for the index of moderated mediation (0.219) was also entirely above zero (0.008-0.458), establishing that knowledgeability positively moderates the mediation of the perceived utility of the unclear product attribute on the evaluation of an ambiguous product offering. We obtained a similar structure of results, although with less statistical power, when the prototype condition was the control.

\section{Effect of prototype-based and goal-based categorization on evaluation across ambiguity conditions (Replication study of Study 2)}

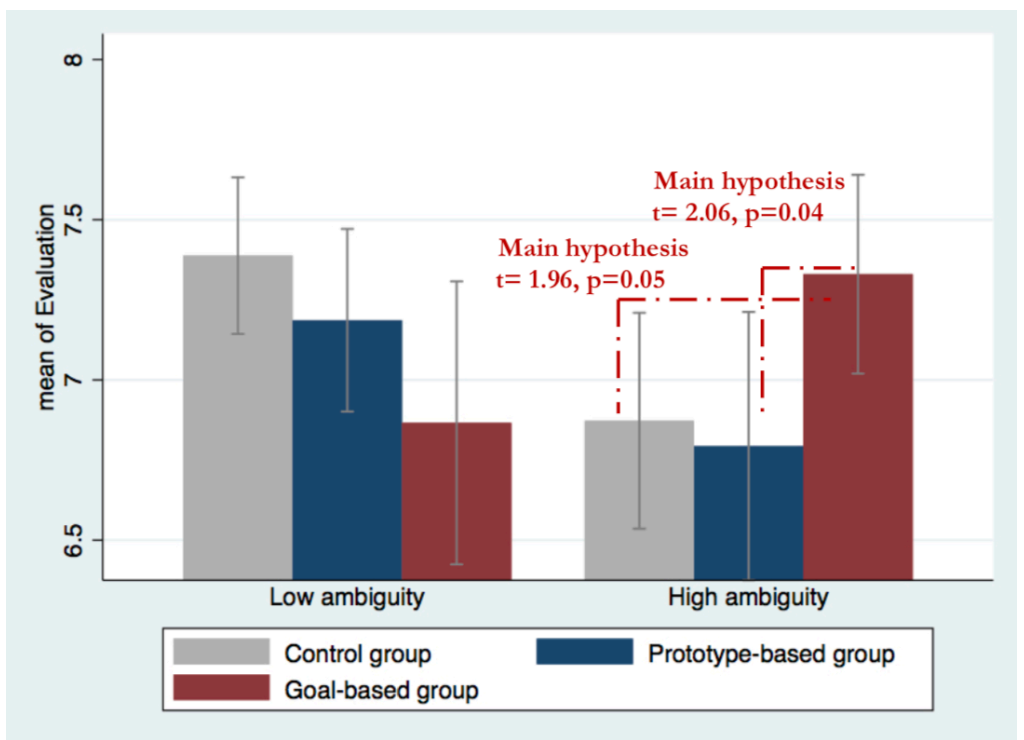

Note: The tests for H1 are shown graphically. H1 is supported because in the high-ambiguity condition, the mean of evaluation is higher in the goal-based group relative to the other two groups $\left(\mathrm{M}_{\mathrm{Goal}}=7.33, \mathrm{SD}=1.15\right.$ vs. $\mathrm{M}_{\text {control }}=$ $6.87, \mathrm{SD}=1.27 ; t(1,106)=1.96, \mathrm{p}=$ 0.05 , two-tailed test and $\left(\mathrm{M}_{\mathrm{Goal}}=7.33\right.$, $\mathrm{SD}=1.15$ vs. $\mathrm{M}_{\text {prototype }}=6.79, \mathrm{SD}=$ 1.44; $\mathrm{t}(1,97)=2.06, \mathrm{p}=0.04$, two-tailed test). 


\section{Mediation via Perceived Utility of the Unclear Attribute (Replication study of Study 2)}

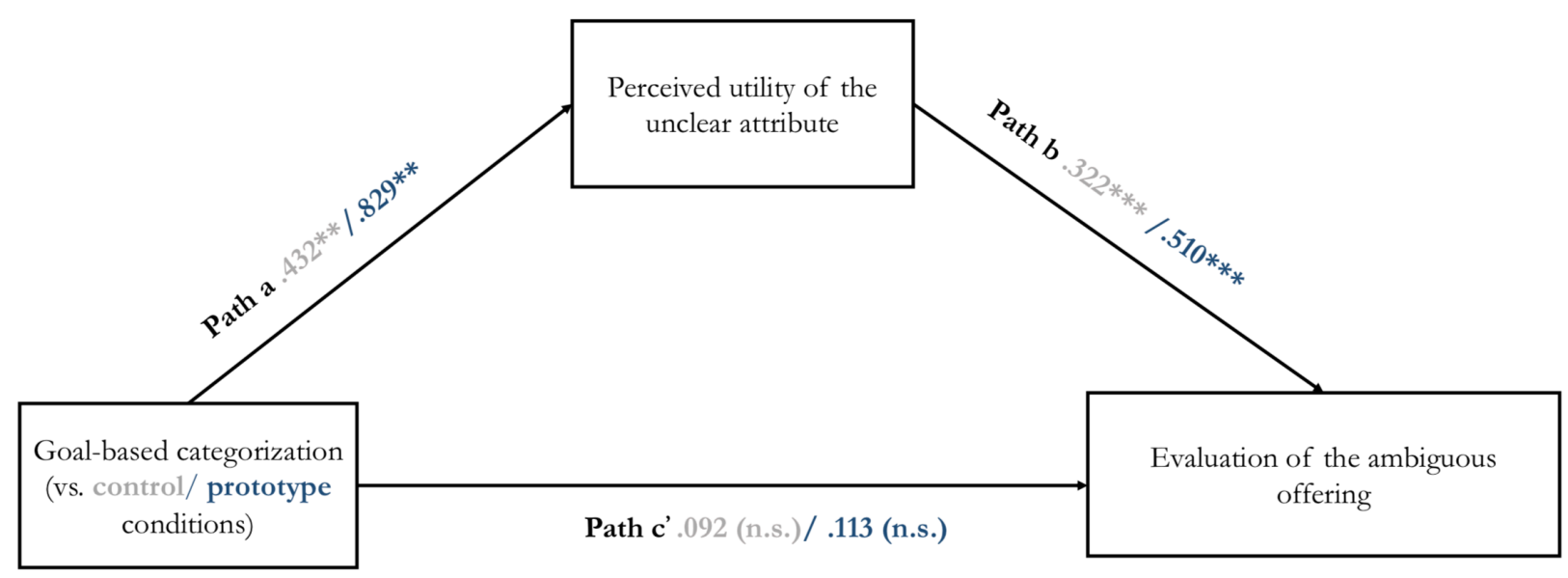

NOTE. ${ }^{*} \mathrm{p}<.05 ;{ }^{* *} \mathrm{p}<.01 ; * * * \mathrm{p}<.001$.

Note: The figure shows that priming goal-based categorization (relatively to being placed in a control condition or being primed on prototype-based categorization) leads to increase the perceived utility of the unclear attribute (path a: beta $_{1}=$ $0.432, \mathrm{p}=0.01, \mathrm{CI}[0.08 ; 0.76]$; beta $2=0.829, \mathrm{p}=0.01, \mathrm{CI}[0.17 ; 1.48])$, which in return positively impacts the evaluation of the ambiguous offering (path $b$; beta $1=0.322, p=0.000, C I[0.21 ; 0.44]$; beta $_{2}=0.510, p=0.000, \mathrm{CI}$ $[0.39 ; 0.63])$. The mediation is strong enough to cancel out the direct effect of goal-based categorization on the evaluation of the ambiguous offering. 